\title{
Identity and semantic negative priming in rapid serial visual presentation streams
}

\author{
Lin $\mathrm{Li}^{1} \cdot$ Ewald Neumann ${ }^{1} \cdot$ Zhe Chen $^{1}$
}

Published online: 5 June 2017

(C) The Psychonomic Society, Inc. 2017

\begin{abstract}
In selective attention tasks, the efficiency of processing concurrently presented target and distractor stimuli in a given display is often influenced by the relationship these stimuli have with those in the previous display. When a to-beattended target on a current trial (the probe trial) matches the ignored, non-target distractor on a previous trial (the prime trial), a response to the target is typically delayed compared with when the two stimuli are not associated. This negative priming (NP) phenomenon has been observed in numerous studies with traditional NP tasks presenting the target and distractor simultaneously in both the prime and probe trial couplets. Here, however, in four experiments using a mixture of stimulus types (letters, digits, English number words, and logographic Chinese number words), target and distractor stimuli were temporally separated in two rapid serial visual presentation (RSVP) streams instead of concurrently presented. The findings provide a conceptual replication and substantial extension of a recent study by Wong (Plos One, 7, e37023, 2012), and suggest that active suppression of irrelevant distracting information is a more ubiquitous form of cognitive control than previously thought.
\end{abstract}

Keywords Selective attention - Negative priming - RSVP . Inhibition $\cdot$ Cognitive control

The everyday world contains a myriad of visual objects that compete for attention. Given the continuous flux of visual information in everyday life, some form of attentional

Ewald Neumann

ewald.neumann@canterbury.ac.nz

1 Department of Psychology, University of Canterbury, Christchurch, New Zealand 8140 selection is often required. Attentional selection abilities are especially pertinent in situations involving conflicting stimuli wherein transiently designated target information is in conflict with rival non-target information. How the human information processing system overcomes attentional competition generated by conflicting stimuli has become a topic of increasingly intense research focus involving a variety of research methods. For instance, Cerf and colleagues have recently conducted brain cell recording research in humans using a Strooplike task (Stroop, 1935) that has begun to address the neural substrates underlying such conflict resolution between concurrently presented overlapping target and non-target objects (Cerf, Thiruvengadam, Mormann, Kraskov, Quiroga, Koch, \& Fried, 2010). Their findings strongly suggest that participants control those neurons representing the targeted object independently of those representing the non-target object, and they do this by enhancing the firing of the neurons that have a preference for the target, while actively inhibiting or suppressing those that encode the non-target.

Cerf et al.'s (2010) study marks a watershed moment for selective attention research. Perhaps most significantly, it helps corroborate decades of research findings and theoretical work on the part of cognitive psychologists who have posited that distractor inhibition and target activation play equally important roles in resolving conflict between competing stimuli for selection (e.g., Neill, 1977; Neumann \& Deschepper, 1991; Tipper, 1985). The enticing clue from the Cerf et al. biophysiological standpoint substantiates the involvement of active suppression of the distractor representation in resolving Stroop-like conflict in selective attention tasks (Chen, 2003; Schooler, Neumann, Caplan, \& Roberts, 1997a, 1997b). More specifically, dissociated neural responses of neural ensembles encoding concurrently overlapping target and distractor stimuli were characterized by distinctly different neural dynamics. Neurons that had a preference for a current target object 
showed heightened activity, whereas those neurons that had a preference for the current non-target distractor stimulus were actively suppressed, but in an original informative manner. In particular, there was no mere reduction in their firing rate. Moreover, their firing rate did not merely reduce to spontaneous baseline rates of firing when the preferred stimulus is not present. Instead, while the competition between the current target and distractor stimulus was being resolved, the firing rate of the neurons with a preference for the distractor reduced their firing rate to below that spontaneous baseline rate. This provides a first order or proximal causal mechanism underpinning conflict resolution, as well providing a mechanism that could lead to longer-term consequences of such distractor inhibition. As such, the active suppression of a conflicting distractor representation may therefore constitute the proximal root cause of the phenomenon known as negative priming (NP), as follows.

In the traditional NP paradigm, participants see two sequentially presented displays: a prime display followed by a probe display, each consisting of a target and a distractor. In one condition, the ignored repetition condition (IR), the target in the probe display is the distractor in the prime display. In another condition, the neutral condition (Control), the target in the probe display is a new stimulus that does not appear in the prime display. Responses to the target in the probe display are typically slower or more error-prone in the ignored repetition condition than in the neutral condition, demonstrating the NP effect. The NP effect indicates that a successful prime selection involves the processing of the distractor to the extent that it can produce a reaction time (RT) cost upon subsequent presentation as a target (Neumann \& Deschepper, 1991; Tipper \& Driver, 1988).

Different stimuli have been used in NP tasks, as well as different manipulations involving the conceptual relationship between the non-target distractors and their subsequent presentation as a target (see Frings, Schneider, \& Fox, 2015, for a review). These may vary from identity (e.g., ignoring a prime distractor letter " $\mathrm{A}$ " that becomes the subsequent probe target letter "A") to various forms of semantic relationships, such as ignoring the picture of a foot and responding subsequently to the word "hand" (e.g., Tipper, 1985; Tipper \& Driver, 1988), or ignoring the word "DOG" and responding next to the word "perro", which is the Spanish translation of the English word "dog" for English-Spanish bilinguals (Neumann, McCloskey, \& Felio, 1999).

The experiments reported in the present article investigate NP using a recently developed paradigm by Wong (2012). Instead of concurrently presented target and non-target stimuli in the prime display, followed by concurrently presented target and non-target stimuli in the probe display, Wong combined the traditional NP paradigm with rapid serial visual presentation (RSVP) typically used in studies that explore the temporal limitation of attentional selection (Dux \& Marios, 2009;
Raymond, Shapiro, \& Arnell, 1992). In the new RSVP-NP paradigm, the prime and probe trial each consists of a stream of stimuli presented sequentially in rapid succession at the same location, and the relationship between the distractor in the prime trial and the target in the probe trial is systematically manipulated. Because the prime distractor and the probe target appear at the same spatial location, this paradigm allows researchers to study how target selection is accomplished when the target and distractor overlap spatially but are separated temporally.

Before elaborating on our adaptation of RSVP in the context of a NP manipulation, we first discuss RSVP and attentional blink $(\mathrm{AB})$ phenomena in the next section. Potential parallels between $\mathrm{AB}$ and the present NP findings will then be returned to in the General Discussion section.

\section{RSVP and attentional blink (AB) phenomena}

One of the most intensively researched phenomena using RSVP procedures is the $\mathrm{AB}$. In the standard $\mathrm{AB}$ task, two target stimuli are presented in close temporal proximity amongst a rapid serial stream of non-target stimuli presented in the same spatial location typically for about $100 \mathrm{~ms}$. Memory recall of the two target stimuli is then required at the end of the RSVP stream. This leads to a period of attenuated accessibility of the second target (T2), following identification of the first target (T1), as long as there is an interven- ing non-target item, and the T2 appears within 200-500 ms after the onset of $\mathrm{T} 1$ in the original stream. Hence, the dependent variable of interest is the accuracy of reporting $\mathrm{T} 2$, conditional on the correct reporting of T1. The so-called "attentional blink" is generally attributed to some form of depleted or disrupted attention to the second target. More specifically, when two targets appear in close temporal proximity, the second target (T2) suffers due to limited attentional resources which are first devoted to processing $\mathrm{T} 1$. The $\mathrm{AB}$ purportedly demonstrates a limit in human attentional processing capacity (Chun \& Potter, 1995; Dux, Coltheart, \& Harris, 2006). Interestingly, if the second appearing target in the stream appears immediately after T1, and within $100 \mathrm{~ms}$, the usual T2 impairment in recall is not observed. This is called lag 1 or $\mathrm{T} 1+$ 1 sparing, as there is no $\mathrm{AB}$ deficit in this instance. The peak $A B$ recall deficit tends to occur when $T 2$ is in either the $T 1+2$ or $\mathrm{T} 1+3$ position when they are within the 200 - to $500-\mathrm{ms} \mathrm{AB}$ time window (see Dux \& Marois, 2009, for a review).

Research has shown that $\mathrm{T} 2$ items presented within the $\mathrm{AB}$ window are nevertheless processed to relatively high levels, even in the event of failure to recall them. This degree of T2 stimulus processing during the $\mathrm{AB}$ has been inferred from priming effects from ostensibly "blinked" stimuli. Numerous studies have shown that a missed target in the $\mathrm{T} 2$ position can nonetheless positively prime a subsequent item that shares the 
same identity or is semantically associated with it (e.g., Luck, Vogel, \& Shapiro, 1996; Maki, Frigen, \& Paulson, 1997; Martens, Wolters, \& van Raamsdonk, 2002; Pesciarelli, Kutas, Dell'Acqua, Peressotti, Job, R., et al., 2007; Shapiro, Driver, Ward, \& Sorensen, 1997).

Harris and colleagues (Harris, Benito, \& Dux, 2010; Harris \& Little, 2010) have shown that distractors from non-target categories presented within the 200- to 500-ms AB window, which might be expected to receive even less attention than a T2 within that time frame, have also been shown to positively prime associated targets. One thing these and the above studies clearly show is that sematic information is being accessed from "blinked" stimuli, otherwise they would not produce such positive priming effects. However, it is not evident whether such RSVP tasks that track the fate of "blinked" stimuli and produce positive priming effects, are suitable for comparison with the current series of experiments, due to methodological differences.

For example, a major difference from standard AB tasks is that in the current experiments there is no requirement to hold two target stimuli in memory and to have memory accuracy assessed for those targets. Instead, ours is a speeded RT experiment with only one target designated by category (e.g., digit vs. letters) in each of two RSVP streams, and this target stimulus can be either preceded or succeeded by a red nontarget stimulus in the same category as the target (e.g., digit). It is interesting to note that in half of the trials in each of the present experiments, the red non-target would consistently fall in the $\mathrm{T} 1+2$ position (and with an onset delay of $234 \mathrm{~ms}$ from T1) in the first RSVP stream, which places it in the position that normally induces the largest amounts of AB (see Dux \& Marois, 2009). One might speculate that at least some of the non-targets in that position might undergo an $\mathrm{AB}$. If such nontargets nevertheless produce semantic negative priming effects, it would corroborate previous evidence that conceptual processing of the non-target distractor is occurring, but it would do so by a negative priming effect, rather than a positive priming effect. We will return to this issue in the General discussion section in view of an inherent limitation in $\mathrm{AB}$ tasks that was pointed out in the review by Dux and Marois, "...because it relies on accuracy rather than RT as a measure of performance, it is difficult to temporally pinpoint the different stages of processing that take place during that task and to identify which of these stages are the loci of interference in dual-target paradigms" [p. 18].

\section{NP under Rapid Serial Visual Presentation (RSVP-NP)}

In Wong's (2012) study, participants saw two streams of stimuli, each with a symbol to indicate the beginning and the end of the stream. Within each stream, five alphanumeric stimuli were presented. Three of them were digits, and they were designated as fillers. The other two were letters, one being the target and the other the distractor. Whereas the distractor had a unique color, the rest of the stimuli all had the same color. The temporal location of the target was unpredictable, and it could appear either before or after the distractor, with one filler item in between. The probe target was either identical or unrelated to the prime distractor, and the participant's task was to make a speeded response to the identity of the target letter. A robust RT cost in the ignored repetition condition was found regardless of whether the distractor appeared before or after the target, and the magnitude of the effect was similar between the two types of trials. These results are largely similar to what one would expect to find in studies that use the traditional NP paradigm, suggesting that the underlying mechanisms may be similar in the two paradigms with respect to interference control.

Because Wong's (2012) paradigm is relatively new and the only prime distractor - probe target relationship he investigated was one of identity (e.g., ignoring a prime non-target letter " $\mathrm{B}$ " that becomes the probe target letter " $\mathrm{B}$ " in the ignored repetition condition), it is important that his findings can be conceptually replicated and extended. Compared to a typical NP experiment, an RSVP-NP experiment places greater demands on participants' attentional system due to the temporal constraints in human information processing. Prior research has shown that these temporal limits can impair stimulus detection, identification, and recall due to problems in central bottleneck (Raymond et al., 1992; Wong 2002), token individuation (i.e., failure to individuate physically identical or similar stimuli as different items; Kanwisher, 1987; Wong \& Chen, 2009), and very short-term memory consolidation (Potter, 1993) . Given these inherent problems in processing RSVP stimuli, it is not obvious that identity NP, and especially semantic NP, would be found in an RSVP-NP paradigm. A conceptual replication would be satisfied if Wong's findings, using letters of the alphabet as stimuli, would generalize to using numerals in an identity NP manipulation. An extension of Wong's finding would be satisfied if the relationship between the prime distractor and probe target in the ignored repetition condition also conformed to a variety of semantic relationship manipulations, just as in more traditional NP tasks (e.g., Driver \& Tipper, 1989; Neumann et al., 1999). Confirming the latter would help support the idea that the same mechanisms involved in resolving concurrent conflict between stimuli are also the mechanisms involved in resolving conflict between temporally separated stimuli.

\section{Overview of the present experiments}

The goal of Experiment 1 was to replicate Wong's (2012) study, but use digits as targets and distractors and letters as 
fillers, in a reversal of Wong's designations. As in Wong's study, the probe target was either identical to or different from the prime distractor. Based on Wong's results, a significant identity NP effect was predicted.

Experiment 2 investigated NP when the probe target differed from the prime distractor in physical form but not in meaning. As in Experiment 1, the target and distractor were both digits in the probe trial, but they were both English number words in the prime trial. If NP was observed again, this would indicate that the prime distractor was inhibited or suppressed at a conceptual or semantic level.

In Experiments 1 and 2, the target and distractor within each trial were either two digits or two number words. In Experiment 3, this was changed. The target and distractor were shown in two different representational forms (digits and logographic Chinese number words). In the prime trial, the distractor was a Chinese number word, but the target was a digit. In the probe trial, the distractor was a digit, but the target was a Chinese number word. This would require participants to shift between two different representational forms (i.e., digit, and logographic Chinese number word) within each trial. If NP was found, this would provide the first evidence showing NP under RSVP with logographic symbols.

Experiment 4 used a cross-language manipulation with Chinese-English bilinguals. A new factor was also introduced. In half the trials, the language 1 (L1) to language 2 (L2) trials, the prime distractor was a Chinese number word while the probe target was its English translation equivalent. In the other half of the trials (the L2-to-L1 trials), the prime distractor was an English number word while the probe target was its Chinese translation equivalent. Experiment 4 thus enabled the investigation of bilinguals' visual-linguistic interference control mechanisms.

\section{Experiment 1}

Experiment 1 used an RSVP paradigm modelled after Wong (2012, Experiment 1). The goal was to replicate the results of Wong using a paradigm similar to his. As in his experiment, participants saw a series of rapidly presented single letters and digits in each trial, and the task was to respond to an alphanumeric target while ignoring the other stimuli. In the IR condition, the probe target had the same form as that of the prime distractor, which was a color singleton. In the control condition, the two stimuli had different forms. Unlike Wong's study, in the present experiment, the target and distractor were digits instead of letters; the neutral stimuli were letters instead of digits; and the target could only appear at one of two instead of one of three temporal positions. Despite these methodological differences, we predicted a significant NP effect on the basis of Wong's results.

\section{Method}

\section{Participants}

Forty-three volunteers, aged from 18 to 40 years, were recruited. Some of them $(n=20)$ were native Chinese speakers who could speak English, and their participation was compensated with either a NZ\$15.00 $(n=8)$ or a NZ\$10.00 $(n=12)$ voucher depending on whether or not they submitted their IELTS scores and participated in a language background survey. ${ }^{1}$ The rest of them were undergraduate students (the majority being non-Chinese) recruited from the participant pool of the University of Canterbury Psychology Department. They took part in the experiment for course credits. All the participants were naïve to the purpose of the experiment, and they reported to have normal or corrected to normal vision.

\section{Apparatus and stimuli}

A 19-in. Philips LCD monitor, driven by a Linux laptop computer (Torvalds, 1997), presented all the stimuli at the refresh rate of $60 \mathrm{~Hz}$. Psychopy (Peirce, 2007), an open source software package, was used to present stimuli and collect responses.

The stimuli were presented in an RSVP paradigm. Each RSVP stream began with the symbol “@”, followed by three uppercase letters intermixed with two Arabic digits, and ended with the symbol "\#" (see Fig. 1). Of the five alphanumeric stimuli, the two digits, one the target and the other the critical distractor, always appeared in the third and fifth positions, and they were equally likely to be " 3 ", " 4 ", " 6 ", or "7". The three letters, which were neutral stimuli and were equally likely to appear in the first, second, and fourth position, were randomly selected from the set "A, B, E, F, G, H, J, K, M, N, P, R, S, W, $\mathrm{X}, \mathrm{Y}$, and $\mathrm{Z}$ ". All the stimuli were written in the Arial font and presented at the center of the screen on a gray background. At a viewing distance of approximately $60 \mathrm{~cm}$, each alphanumeric stimulus subtended a visual angle of $0.96^{\circ} \times 0.57^{\circ}$.

The stimuli were all black except for one of the two digits, which was red. This red singleton was equally likely to appear in the third or fifth position in both the prime and probe trials, with the black digit in the other position not occupied by the distractor.

\section{Design and procedure}

The experiment used a within-participants design. The principal manipulations were the prime-probe relationship (i.e., the

\footnotetext{
${ }^{1}$ IELTS stands for International English Language Testing System, which is an international standardised English language proficiency examination. The IELTS data were collected for another series of experiments that are not reported here.
} 

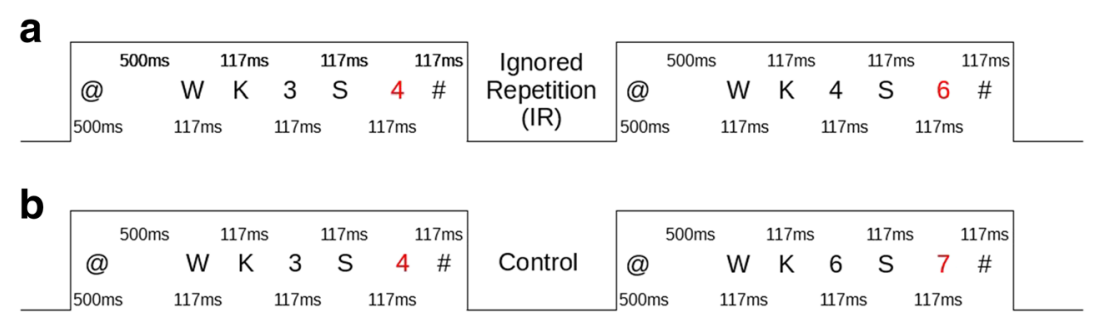

TIME

Fig. 1 Examples of stimulus displays from Experiment 1. The task was to make a speeded response to the identity of the black digit. In the ignored repetition condition (A), the target in the probe trial was the same in form as the distractor in the preceding prime trial. In the control condition $(\mathbf{B})$, the target was a digit not presented in the prime trial
IR condition where the prime distractor and the probe target were identical in form vs. the control condition where they were different), target position in the prime trial (i.e., the prime target before the prime distractor, or Prime T1, vs. the prime distractor before the prime target, or Prime D1), and target position in the probe trial (i.e., the probe target before the probe distractor, or Probe T1, vs. the probe distractor before the probe target, or Probe D1). All these factors were independently manipulated, and the proportion of each type of trial was the same. The latter two factors gave rise to four target/ distractor positions across the prime and probe trials. In the Prime T1-Probe T1 condition, the target was at the third position in both the prime and probe trials. In the Prime T1-Probe D1 condition, the target was at the third position in the prime trials but at the fifth position in the probe trials. In the Prime D1-Probe T1 condition, the target was at the fifth position in the prime trials but at the third position in the probe trials. Finally, in the Prime D1-Probe D1 condition, the target was at the fifth position in both the prime and probe trials.

The participants were told to respond to the black digit (the target) as quickly and as accurately as possible by pressing one of four designated keys on a computer keyboard. Each trial began with a central fixation "@” for 500 ms, followed by a 500-ms blank screen. The letters and digits were then presented one at a time for $117 \mathrm{~ms}$ each at the same location in the centre of the screen. Each stream ended with a 117-ms post-mask “\#”, followed by a blank screen until a response was made. Participants pressed one of four labelled keys: "e", "r", "i", and "o" for responses " 3 ", " 4 ", " 6 ", and " 7 ," respectively. The responses were made by the left middle and index fingers for responses " 3 " and " 4 ", and by the right index and middle fingers for responses " 6 " and " 7 ." The experiment consisted of 384 pairs of prime-probe trials divided into four blocks. The participants were encouraged to take a break after each block. No feedback was provided during the experimental session.

Before the experimental session began, there were three practice blocks with 16 prime-probe pairs in each block. In the first two blocks, there was immediate accuracy feedback after each response. In the third block, no feedback was provided. The item presentation duration decreased across the blocks, with $160 \mathrm{~ms} /$ item in the first block, $140 \mathrm{~ms} /$ item in the second block, and $117 \mathrm{~ms} /$ item in the final block. The whole experiment (practice plus the experimental session) took about $45 \mathrm{~min}$ to complete.

\section{Results and discussion}

Seven participants' data were excluded from analyses due to high error rates (>30\%). For the remaining 36 participants, we calculated each person's mean RT and percentage error in the probe trials. Only those probe trials in which both the prime and probe targets were correctly identified and the RT was between $200 \mathrm{~ms}$ and 2,000 ms were included in the calculation of the mean RT. The mean RT for each participant was then converted into the adjusted RT, or AdjRT (AdjRT = RT/(1-\% error)). Because the AdjRT takes into account both response speed and accuracy (Chambers, Stokes, \& Mattingley, 2004; Townsend \& Ashby, 1983), it is a more sensitive measure for processing efficiency than either the mean or median RT, which is prone to speed-accuracy trade-offs.

The AdjRT data are shown in both Fig. 2 and Appendix A, Table 1. The mean RTs and error rates are shown in Appendix A, Table 2. In all the figures and tables that depict the results of the experiments in this paper, the error bars show the withinsubjects standard error of the means (Cousineau, 2005). A $2 \times$ $2 \times 2$ repeated-measures ANOVA was conducted. ${ }^{2}$ A significant NP effect was found, $F(1,35)=8.98, M S E=8463, p=$ $.005, \eta_{\mathrm{p}}{ }^{2}=.20$, indicating slower responses in the IR condition (801 ms) compared with the control condition (768 ms). There was also a significant interaction between prime target position and probe target position, $F(1,35)=13.29, M S E=2697$, $p<.001, \eta_{\mathrm{p}}{ }^{2}=.28$. When the prime target preceded the prime

\footnotetext{
${ }^{2}$ We also conducted analyses on the mean RTs and error rates for all the experiments reported in this study. The pattern of results is consistent with that found in the AdjRT data.
} 


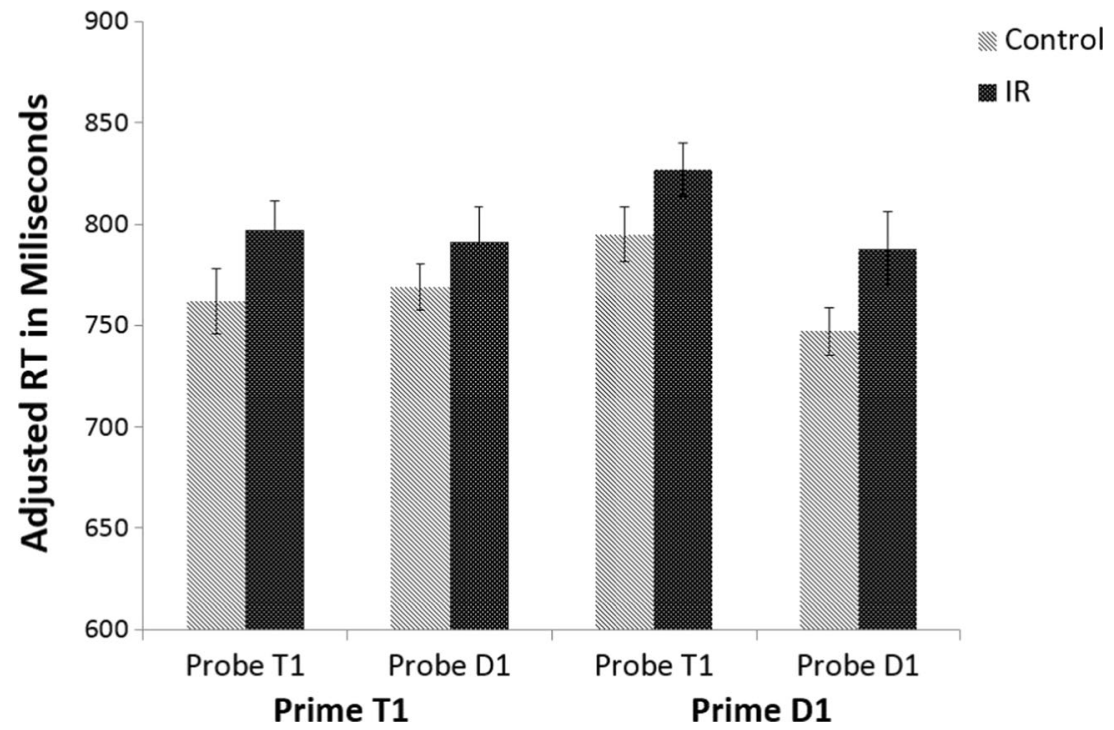

Fig. 2 Results from Experiment 1. T1, the target appeared before the distractor. D1, the distractor appeared before the target. $R T$ reaction time

distractor, responses to the probe target did not differ regardless of its temporal position in the probe trials $(779 \mathrm{~ms}$ and $781 \mathrm{~ms}$ for the Probe T1 and Probe D1 trials, respectively). However, when the prime target followed the prime distractor, responses to the probe target were delayed when it was shown before rather than after the probe distractor $(811 \mathrm{~ms}$ and $768 \mathrm{~ms}$ for the Probe T1 and Probe D1 trials, respectively). Tukey's honestly significant difference (HSD) test further indicated longer RT in the Prime D1-Probe T1 condition than in the other three conditions, with no differences among the latter conditions. No other effects reached significance.

To assess the NP effect as a function of the target/distractor position in the prime and probe trials individually, we conducted four planned $t$ tests for dependent means. A significant NP effect was found in the Prime T1-Probe T1 condition, $t$ (35) $=1.70, p=.049, d=0.29$; in the Prime D1-Probe T1 condition, $t(35)=1.95, p=.030, d=0.32$; and in the Prime D1-Probe D1 condition, $t(35)=2.04, p=.025, d=0.34$. The NP effect was not significant in the Prime T1- Probe D1 condition, $t(35)=1.11, p=.137, d=0.19$. As indicated by the value of $d$ in each condition, the results show that the size of the NP effect was medium in three of the four conditions.

The most important finding of Experiment 1 was the main effect of NP. Although the present experiment differed from Wong's (2012) experiment in the stimuli and the temporal positions of the target/distractor, in both studies the participants took longer to respond to the probe in the IR condition compared with the control condition, demonstrating identity NP. These results show that presenting the target and distractor sequentially among other task irrelevant stimuli in an RSVP paradigm can evoke NP. To our knowledge there is only one other study that used temporally separated, singularly presented stimuli that also showed an identity NP effect (i.e., Neumann \& DeSchepper, 1992). In their study, target relevancy was cued by a shift in the presentation location of stimuli, rendering the prior stimulus an irrelevant distractor. When such a non-target distractor was subsequently presented for a classification judgement, it produced an effect that was interpreted as a traditional identity NP effect. Those results may be seen as consistent with the findings of Wong and the present experiment, thus supporting the contention that the same mechanism(s) responsible for these effects are shared in common.

In addition to the NP effect, stimulus position also affected performance. As shown by the Tukey's HSD test described above, the AdjRT was longer in the Prime D1-Probe T1 condition (i.e., when the prime target was at the fifth position and the probe target at the third position) than in the other three conditions. This result was likely caused by the shorter temporal interval between the responses to the prime and probe targets in the Prime D1-Probe T1 condition compared with the other conditions. When the prime and probe targets were temporally close together, participants did not have sufficient time to replenish the depleted resources used in responding to the prime target before the probe target appeared, resulting in delayed responses.

NP did not interact with stimulus position in either the present experiment or Wong's (2012) study. This indicates that the temporal position of the prime distractor, i.e., whether it appeared before or after the prime target, had negligible effect on the magnitude of NP. The absence of the interaction may be somewhat surprising, given that one might expect a distractor that precedes the target to interfere more than a distractor that follows the target, resulting in stronger inhibition and a larger NP effect (see Wyatt \& Machado, 2013a, 2013b, for evidence of reactive inhibition). Whether the absence of the distractor position effect on NP had something to do with the distractor being a color singleton and/or the rapid presentation rate of the stimuli in the RSVP stream was unclear. 
It is worth noting that two aspects of the data in Experiment 1 differed from those in Wong (2012). First, the magnitude of $\mathrm{NP}$ was larger in his experiment (66 ms in mean RT) than in the present one (17 ms in mean RT and $33 \mathrm{~ms}$ in AdjRT). Second, stimulus position affected performance in Experiment 1, but not in Wong's study. Although it is difficult to know the exact cause(s) for these differences, two factors may have contributed to the observed results in the two studies. One was the temporal position of the target. The target could appear at one of five positions in Wong's study but only one of two positions in Experiment 1 . The greater position uncertainty in Wong could induce participants to apply stronger inhibition to the prime distractor, resulting in larger NP. The other was the type of prime trials excluded in the probe RT analyses. In the present study, a probe trial was included in the RT analyses if and only if both the probe trial and its preceding prime trial were responded to correctly. This was not the case in Wong's study, in which only a portion of incorrect prime trials, i.e., those in which the participants reported the identity of the distractor rather than that of the target in the prime trials, were excluded from probe RT analyses. (Note that pressing the key indicated by the prime distractor was only one of three possible wrong responses a participant could make.) Because RT is typically longer after an incorrect response than after a correct response (Chun \& Wolfe, 1996; Fleck \& Mitroff, 2007), including incorrectly responded prime trials in the analyses of the probe RTs could increase the variability of the RT results. This could reduce the sensitivity of Wong's study in detecting the stimulus position effect.

\section{Experiment 2}

In Experiment 1, participants responded to digits in every trial, and the prime and probe were both Arabic digits. In Experiment 2, whereas the target and distractor were again digits in the probe trial, they were English number words in the prime trials (e.g., "THREE" instead of "3"). Thus, participants would need to switch between two different representational forms between the prime and probe trials. The goal of the experiment was to investigate whether NP would still be found in an RSVP paradigm when the prime and probe were the same in meaning but different in form.

Previous research that used the traditional NP paradigm has reported inconsistent results regarding NP across different representational forms. Tipper and Driver (1988) provided evidence showing that the NP effect can be observed between a pictorial representation of an object (e.g., the picture of a dog) and its corresponding verbal representation (e.g., the word "dog"). Moreover, cross-language NP effects have been found by Neumann et al. (1999). These results show that the NP effect can occur at the semantic level in addition to the physical level (e.g., Fox, 1995; May, Kane, \& Hasher, 1995).
However, there is also evidence showing that NP does not always occur across semantically related but physically different stimuli. MacLeod, Chiappe, and Fox (2002) found no evidence of NP for semantically related words. No NP effects were observed by Lammertyn and Fias (2005) between a verbal prime and a digit probe, either. These and other studies (e.g., Duscherer \& Holender, 2002; Hutchison, 2002; Koelewijn, Van der Burg, Bronkhorst, \& Theeuwes, 2008) suggest that while NP can be obtained reliably when the prime and probe have the same form, the effect is elusive when these stimuli are only semantically related.

With respect to the present experiment, we did not make a priori predictions about the results. On the one hand, it is possible that a significant NP effect would emerge. Previous research has shown that NP is more likely to manifest when target selection is difficult and distractors interfere consistently and strongly with the target in the prime trial (Pritchard \& Neumann, 2009; Tipper \& Cranston, 1985). In the present study, the prime distractor is a color singleton presented at the center of attentional focus. Given the salience of the prime, it would capture attention and undergo substantial processing. To enable target selection, the visual system would need to evoke strong suppression to inhibit the prime or to ignore the prime actively, perhaps by attaching a very salient "not-to-respond" or "unwanted" tag to its representation. In either way, the NP effect should emerge. On the other hand, it is also possible that no NP would be found. Semantic NP in general is not very robust, as evidenced by a number of previous studies that have failed to find a significant effect (e.g., Lammertyn \& Fias, 2005; MacLeod et al., 2002). It is also unclear whether the requirement of switching between two different representational forms would encourage participants to keep both representational forms active, thereby eliminating the NP effect.

\section{Method}

The method was the same as that in Experiment 1 except that the target and distractor in the prime trials were changed from Arabic digits to English number words (i.e. "THREE", "FOUR", "SIX", and "SEVEN") written in the 28-point Arial font. Thus, the task was to identify either the black number word or the black Arabic digit in a trial. The participants pressed the "e" key for either " 3 " or "THREE", the " $r$ " key for " 4 " or "FOUR", the "i" key for " 6 " or "SIX", and the "o" key for "7" or "SEVEN". Twenty-one new undergraduate students from the same participant pool took part in the experiment. ${ }^{3}$ They received either course credits or a NZ $\$ 10$ voucher for their participation.

\footnotetext{
${ }^{3}$ We reduced the number of participants in Experiment 2 in anticipation of the number of participants we were able to recruit in the subsequent experiments, which would require Chinese-English bilinguals.
} 


\section{Results and discussion}

Six participants' data were excluded from analyses due to high error rates (over 30\%). For the rest of the participants, their data were treated in the same way as that described in Experiment 1. The AdjRT data are shown in Fig. 3 and in Appendix A, Table 1. The mean RTs and error rates are shown in Appendix A, Table 2. As in Experiment 1, a $2 \times 2 \times 2$ repeated-measure ANOVA was conducted on the AdjRT data. A significant NP was again found, $F(1,14)=4.77, M S E=$ $1754, p=.047, \eta_{\mathrm{p}}^{2}=.25$, indicating slower responses in the IR condition (799 ms) than in the control condition $(783 \mathrm{~ms})$. The main effects of prime target position and probe target position were also significant, $F(1,14)=9.49, M S E=2635, p=.008$, $\eta_{\mathrm{p}}{ }^{2}=.40$, for prime target position; and $F(1,14)=6.84, M S E$ $=28996, p=.020, \eta_{\mathrm{p}}{ }^{2}=.33$, for probe target position. These results indicate that response latencies were longer when the targets in the prime and probe trials were closer in time than when they were further apart. Specifically, the participants were faster to respond to the probe target when the prime target preceded the prime distractor $(777 \mathrm{~ms})$ rather than followed it $(805 \mathrm{~ms})$, and when the probe target was after the probe distractor $(750 \mathrm{~ms})$ rather than before it $(832 \mathrm{~ms})$. No other effects were significant.

To assess the NP effect as a function of stimulus position, we again conducted $t$ tests for dependent means. A marginally significant NP effect was found in the Prime T1-Probe T1 condition, $t(14)=1.66, p=.059, d=0.43$. No significant NP effect was found in the other three conditions, with $t(14)=$ $1.11, p=.143, d=0.29$ in the Prime T1-Probe D1 condition; $t$ $(14)=1.10, p=.145, d=0.27$ in the Prime D1-Probe T1 condition; and $t(14)=1.34, p=.100, d=0.34$ in the Prime D1-Probe D1 condition.
In Experiment 2, the participants responded to a number word in the prime trial but to an Arabic digit in the probe trial. Although the prime and probe shared little physical resemblance, a significant main effect of NP was found. This result is consistent with previous studies that observed NP across different representational forms (Tipper \& Driver, 1988). It also extended the results of Experiment 1 and Wong's (2012) study by providing evidence that NP could occur at a semantic level in an RSVP paradigm. As far as we are aware, there is only one other study using temporally separated target and distractor stimuli that also showed a semantic NP effect (i.e., Neumann, Cherau, Hood, \& Steinnagel, 1993). In their study, target relevancy was cued by a shift in the presentation location of stimuli, rendering the prior stimulus an irrelevant distractor. For example, if the word "nurse" was an irrelevant non-target distractor and the associated concept "doctor" was subsequently presented for a classification judgement, it produced an effect that was interpreted as a semantic NP effect. Those results may be seen as consistent with the present semantic NP effect in the RSVP task, lending additional support to the contention that the same mechanism(s) responsible for such effects are shared in common.

It is likely that the salience of the prime played an important role in the manifestation of NP in Experiment 2. Unlike the traditional NP paradigm in which the prime distractor is typically shown concurrently with the target and often at a peripheral location, in the present study, the prime was displayed alone at the center of attentional focus. Being a color singleton also ensured that the prime distractor was very salient. Previous research has shown that salient distractors attract attention and evoke strong reactive inhibition (Houghton, Tipper, Weaver, \& Shore, 1996; Wyatt \& Machado, 2013a; 2013b). Hence, the NP effect in the present study.

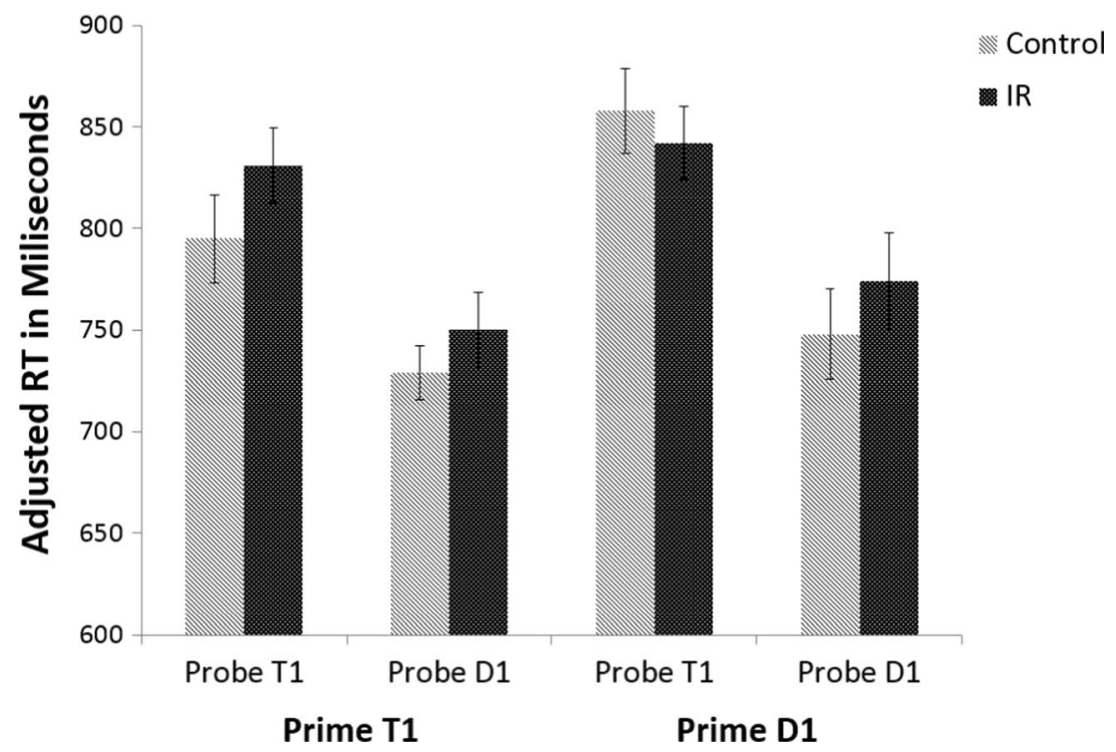

Fig. 3 Results from Experiment 2. T1, the target appeared before the distractor. D1, the distractor appeared before the target. RT reaction time 
Despite the significant main effect of NP, the effect did not reach significance when examined in each individual condition. Of the four combinations of target/distractor locations across the prime and probe trials, only one condition, the condition in which the target appeared before the distractor in both the prime trial and the probe trial (i.e., the Prime T1Probe $\mathrm{T} 1$ condition), showed a marginally significant NP effect. An important feature that distinguished this condition from the others is the absence of any task relevant stimuli (i.e., digits or number words) between the distractor in the prime trial and the target in the probe trial. In other words, because the prime distractor was the only task relevant stimulus before the appearance of the probe target in the Prime T1Probe $\mathrm{T} 1$ condition, the representation of the prime distractor was less likely to be disrupted before the onset of the probe target in this condition than in the other three conditions, resulting in the observed pattern of data in Experiment 2.

Although only the Prime T1-Probe T1 condition showed a marginally significant NP effect, this does not necessarily mean that the overall NP effect in Experiment 2 was smaller than that in Experiment 1. We recruited fewer participants in Experiment 2 than in Experiment 1 in anticipation of the limited number of participants we would be able to recruit in Experiments 3 and 4, which would require Chinese-English bilinguals. The reduction in participant number reduced the sensitivity of the experiment, and this could lead to the non-significant NP effects when individual NP effects in each condition were assessed. This is evidenced by the magnitude of the effect size of NP in Experiment 2 ( $d$ ranged from .27 to .43 ), which was comparable to that of Experiment 1 ( $d$ ranged from .19 to .34).

\section{Experiment 3}

In both Experiment 1 and Experiment 2, the target and distractor were shown in the same representational form within each trial (i.e., both were digits or English number words). In
Experiment 3, they were from two different representational forms. In the prime trials, the distractor was a logographic Chinese number word, but the target was an Arabic digit. In the probe trials, the distractor was an Arabic digit, but the target was a Chinese number word (see Fig. 4). Thus, to respond to the target in the prime and probe trials, participants would have to shift between two different representational forms.

Because shifting between different representational forms could be challenging, participants could choose to keep both representational forms active throughout the experiment instead of inhibiting one representational form in the prime trial and the other one in the probe trial. Although there was no evidence that such a strategy was used in Experiment 2, in which the targets in the prime and probe trials also differed in representational forms, this could be due to the competition between the presentations of the target and distractor, which were written in the same representational form within each trial. Unlike Experiment 2, the target and distractor in Experiment 3 were written in different representational forms within the prime stream and the probe stream, and this may result in less competition between the representations of the target and the distractor in Experiment 3 than in Experiment 2. Should the participants still be induced to inhibit the distractor, this would result in NP. Alternatively, if they were induced to keep both representational forms above the baseline, due to lack of competition, this would lead to null or even positive priming.

\section{Method}

The method was the same as that in Experiment 1 except that the prime distractor and the probe target were both Chinese number words. Thus, instead of " 3 ", " 4 ", " 6 ," or "7", the corresponding Chinese number word “三”, “四”, “六”, or "七” was used, and each was written in red or black depending on whether the stimulus was a prime or probe. At a viewing distance of approximately $60 \mathrm{~cm}$, each Chinese number word
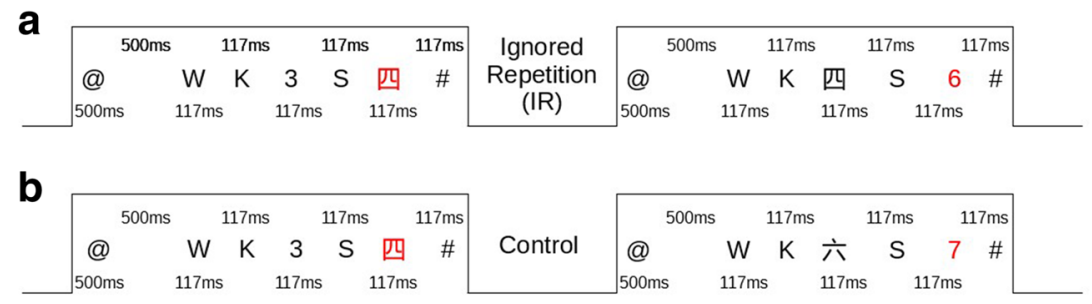

TIME

Fig. 4 Examples of stimulus displays from Experiment 3. The task was to identify the black Arabic digit in the prime trial but to identify the black number word in the probe trial. In the ignored repetition condition $(\mathbf{A})$, the target in the probe trial was the same in form as the distractor in the preceding prime trial (A). In the control condition, the target was a digit not presented in the prime trial 
was presented with the same font setting that matched the size of the other stimuli.

As shown in Fig. 4, the target was an Arabic digit and the distractor a Chinese number word in the prime trial and this was reversed in the probe trial. The participants pressed the "e" key for either “ 3 " or “三”, the " $\mathrm{r}$ " key for “4” or “四”, the " $\mathrm{i}$ " key for “6" or “六”, and the “o" key for “7” or “七”. Twenty-two Chinese-English bilinguals took part in the experiment. Each was paid NZ $\$ 10.00$ for their participation. All the other aspects of the experiment were identical to those in Experiment 1.

\section{Results and discussion}

The data were treated in the same way as in Experiment 1. Five participants' data were excluded because of high error rate (over 30\%). For the rest of the participants, the AdjRT data are shown in Fig. 5 and in Appendix A, Table 1; and the mean RTs and error rates are shown in Appendix A, Table 2. A $2 \times 2 \times 2$ repeated-measure ANOVA on the AdjRT data was conducted. Once again, there was a significant main effect of $\mathrm{NP}, F(1,16)=6.66, M S E=1609, p=.020, \eta_{\mathrm{p}}{ }^{2}=.29$, indicating slower responses in the IR condition (714 ms) than in the control condition $(696 \mathrm{~ms})$. There was also a significant interaction between prime target position and probe target position, $F(1,16)=16.88, M S E=2655, p<.001, \eta_{\mathrm{p}}{ }^{2}=$ .51 . This result shows that when the prime target appeared before the prime distractor, participants' response latencies to the probe target did not differ regardless of its position in the probe trial $(704 \mathrm{~ms}$ in the Probe T1 condition and $700 \mathrm{~ms}$ in the Probe D1 condition). However, when the prime target appeared after the prime distractor, the participants took substantially longer to respond to the probe target when it preceded the probe distractor $(746 \mathrm{~ms})$ rather than when it followed the probe distractor $(669 \mathrm{~ms})$. Once again, Tukey's HSD test showed longer RT in the Prime D1-Probe T1 condition than in the other three conditions, with no differences among the latter group. No other effects reached significance.

Individual $t$ tests were again conducted to assess the NP effect as a function of the target/distractor position in the prime and probe trials. As in Experiment 2, a marginally significant $\mathrm{NP}$ effect was found in the Prime T1-Probe T1 condition, $t$ (16) $=1.40, p=.090, d=0.34$. No significant effects were found in any of the three other conditions, with $t(16)=1.11, p=.142, d$ $=0.27$ in the Prime T1-Probe D1 condition; $t(16)=1.26, p=$ $.113, d=0.30$ in the Prime D1-Probe T1 condition; and $t(16)=$ $0.44, p=.333, d=0.10$ in the Prime D1-Probe D1 condition.

In both Experiment 1 and Experiment 3, the prime distractor and the probe target were the same in form. However, unlike Experiment 1, in which the participants responded to an Arabic digit in every trial, the participants in Experiment 3 had to switch between digits and Chinese number words across any two consecutive trials and the target and distractor differed in form within a trial. Despite these differences, the results of Experiment 3 were remarkably similar to those of Experiment 1. In both experiments, a significant main effect of NP was found, as was the interaction between prime target position and probe target position. These results indicate that shifting between different representational forms did not induce participants to keep both representational forms active. Instead, the distractor was actively ignored or inhibited. It is likely this response strategy was adopted because the distractor was extremely salient in the present study. The strong bottom-up activation generated by the onset of the distractor would make its representation highly competitive relative to the representation of the target. Keeping both representational forms active across trials would likely impair

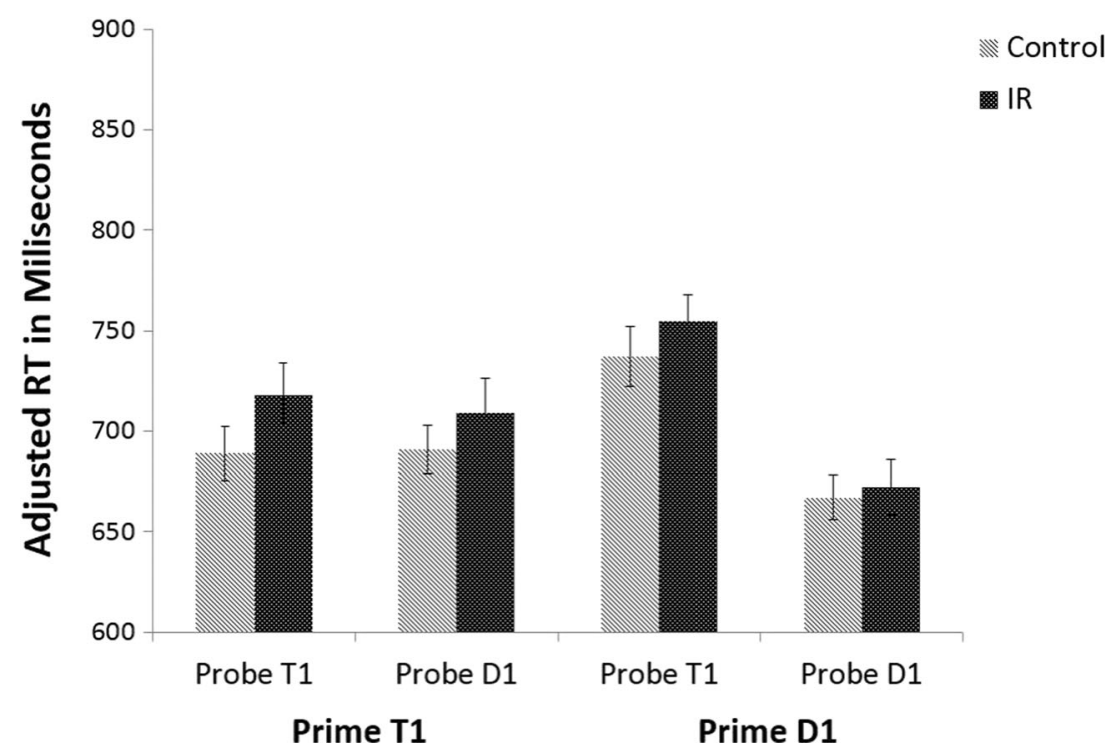

Fig. 5 Results from Experiment 3. T1, the target appeared before the distractor. D1, the distractor appeared before the target. $R T$ reaction time 
target selection on a given trial, prompting the visual system to evoke inhibition or to place a "do-not-respond" tag to the distractor representation.

\section{Experiment 4}

Experiment 3 established that Chinese bilingual participants could shift quickly from responding to an Arabic digit in the prime trial to responding to a Chinese number word in the probe trial and still show NP when the prime and probe were the same Chinese number word. In Experiment 4, participants again responded to an Arabic digit in the prime trial and to a number word in the probe trial. However, the prime distractor differed from the probe target. In half of the trials, the prime distractor was a Chinese number word (e.g., "三”) and the probe target was the corresponding word written in English (e.g., "THREE"). In the other half of the trials, the two stimuli switched their roles such that the prime distractor was an English number word and the probe target its Chinese counterpart. The goal of the experiment was twofold: to test semantic NP across two different languages, and to provide converging evidence for the distractor inhibition account of NP.

Previous research on bilingual language switching in naming tasks has shown that the cost of language switching from one language to another differs depending on whether participants switch from their dominant language (L1) to a weaker language (L2) or vice versa (e.g., Filippi, Karaminis, \& Thomas, 2014; Macizo, Bajo, \& Paolieri, 2012; Meuter \& Allport, 1999). Importantly, switching cost is larger when participants switched from L2 to L1 than from L1 to L2, and this asymmetry is explained in terms of stronger inhibition that participants have to impose on L1 when they engage in L2 due to the higher baseline activation level of L1 than L2 (Meuter \& Allport, 1999). Similar asymmetric switching cost has also been found in other tasks of unequal strength that do not involve language switching (e.g., De Jong, 1995; Harvey, 1984), indicating that switch-cost asymmetry is a general phenomenon not limited to the domain of language switching in bilinguals. Furthermore, using a NP paradigm with bilingual participants, Fox (1996) observed semantic cross-language NP when the prime distractor was in L1 and the probe target was in L2, but not vice versa. In addition, in a subsequent experiment in which the prime distractor and the probe targets were translational equivalents, the participants showed a larger NP effect in the L1to-L2 trials than in the L2-to-L1 trials.

In light of the results of these and other related studies (see Kiesel, Steihauser, Wendt, et al., 2010, for a review), we hypothesized that the participants in Experiment 4 would show different degrees of NP depending on the languages of the prime and probe. Specifically, NP would be stronger when the prime distractor was written in Chinese and the probe target in English (i.e., L1-to-L2 trials) than when it was the other way around (i.e., L2-to-L1 trials). This is because Chinese was the participants' dominant language, and compared with English, a distractor written in Chinese should evoke stronger inhibition when it was the prime distractor.

The above hypothesis was based on the assumption that the distractor in the prime trial would be inhibited. However, in addition to the inhibition-based account of NP, NP has also been explained in terms of non-inhibitory processes (see D’Angelo, Thomson, Tipper, \& Milliken, 2016; Frings et al., 2015; Mayr \& Buchner, 2007; and Tipper, 2001, for reviews), with the most influential account being the episodic retrieval theory proposed originally by Neill and colleagues (Neill \& Valdes, 1992; Neill, Valdes, Terry \& Gorfein, 1992). According to these researchers, when a stimulus is encountered, it automatically triggers the retrieval of the memory trace of the most recent episode associated with that stimulus including its response tag. In the NP paradigm, the probe target is identical or semantically related to the prime distractor in the IR condition. Because the probe target requires a response but the memory trace associated with the prime distractor contains a "do-not-respond" tag, this creates a conflict. Resolving the conflict requires time, resulting in NP.

It is important to note that while the distractor inhibition and episodic retrieval accounts of NP make the same predictions in most NP experiments, they make different predictions in Experiment 4. Whereas the distractor inhibition account predicts different degrees of NP depending on the languages of the prime and probe, the episodic retrieval theory predicts equivalent degree of NP regardless of the languages of the stimuli. Thus, if the participants showed stronger NP in the L1-to-L2 trials than in the L2-to-L1 trials, this result would provide support for the distractor inhibition theory of NP.

\section{Method}

The method was the same as that of Experiment 3 except for the following changes. In addition to the three factors used in the previous experiments (i.e., prime-probe relation, or IR vs. Control; prime target position, or Prime T1 vs. Prime D1; and probe target position, or Probe T1 vs. Probe D1), a new factor was introduced (see Fig. 6). The new factor was the languages of the prime and probe, or Language (i.e., from prime distractor in Chinese to probe target in English vs. from prime distractor in English to probe target in Chinese, or L1-to-L2 vs. L2-toL1). Thus, the design of the experiment was a $2 \times 2 \times 2 \times 2$ within-subjects design. All the factors were manipulated independently. There were as many L1-to-L2 trials as there were L2-to-L1 trials, and the two types of trials were presented randomly within a block. Participants responded to a different representational form on each trial, and depending on the specific experimental condition, the target could be an Arabic digit, 


\section{a}

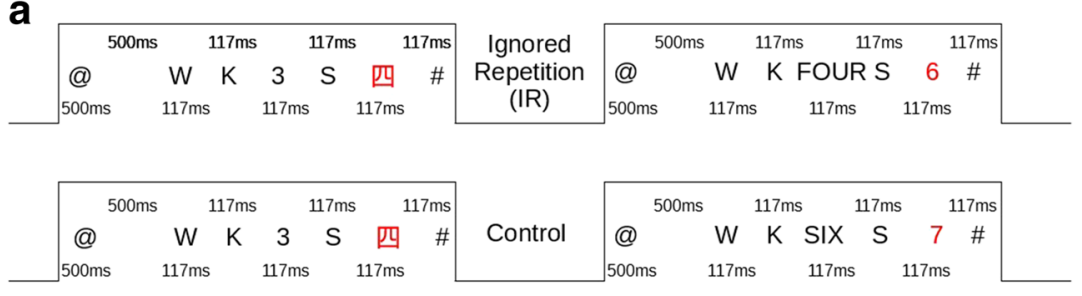

TIME

\section{b}

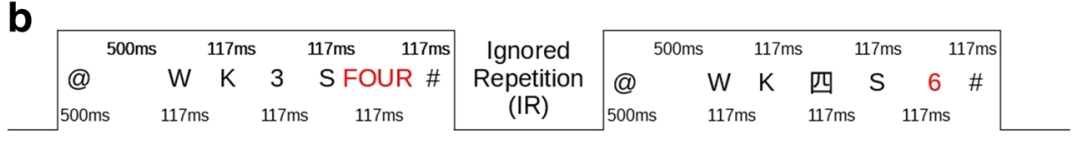

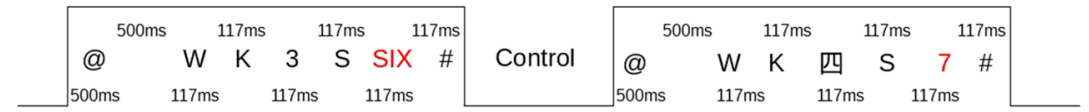

TIME

Fig. 6 (A and B) Examples of stimulus displays from Experiment 4, with the L1-to-L2 trials (A) shown separately from the L2-to-L1 trials (B). In the L1-to-L2 trials, the distractor in the prime trials was written in the participants' first language while the target in the probe trials was written

a Chinese number word, or an English number word. The "e" response key was used for responses " 3 ", "THREE", or “三”; the "r" key for "4", "FOUR", or “四”; the "i" key for " 6 ", "SIX", or “六”; and the "o" key for “7”, "SEVEN", or “七”. Twenty-two new Chinese-English bilinguals took part in the study. As before, none of them knew the purpose of the study.

\section{Results and discussion}

The data were treated in the same way as in the previous experiments. Seven participants' data were excluded because of high error rate (over 30\%). For the rest of the participants, the AdjRT data are shown in Figs. 7A and B and in Appendix B, Table 3, with Fig. 7A depicting the results of the L1-to-L2 trials and Fig. 7B the results of the L2-to-L1 trials. The mean RTs and error rates are shown in Appendix B, Table 4.

A $2 \times 2 \times 2 \times 2$ repeated-measures ANOVA was conducted on the data. The main effect of language was significant, $F(1$, 14) $=9.44, M S E=5644, p=.008, \eta_{\mathrm{p}}{ }^{2}=.40$. Reponses were faster in the L1-to-L2 trials $(675 \mathrm{~ms})$ than in the L2-to-L1 trials (705 ms). The main effect of prime target position was also significant, $F(1,14)=6.55, M S E=4171, p=.023, \eta_{\mathrm{p}}{ }^{2}=$ .32 , indicating faster responses when the prime target was shown before the prime distractor (679 ms) compared with when their temporal positions were switched (701 ms). In addition, there was a marginally significant interaction between Language and prime-probe relation, $F(1,14)=4.19, M S E=$ in the participants' second language. In the L2-to-L1 trials, the distractor in the prime trials was written in the participants' second language while the target in the probe trials was written in the participants' first language

$1969, p=.060, \eta_{\mathrm{p}}^{2}=.23$, suggesting a larger NP effect in the L1-to-L2 trials $(18 \mathrm{~ms})$ than in the L2-to-L1 trials $(-5 \mathrm{~ms})$.

Although the Language by prime-probe relation interaction was only marginally significant, from a theoretical perspective, it is important to examine the NP effect in the L1-to-L2 condition and the L2-to-L1 condition separately. As we described earlier, previous research has shown that switching cost for bilinguals is generally larger when they have to inhibit L1 (Filippi et al., 2014; Macizo et al., 2012; Meuter \& Allport, 1999). Neumann et al. (1999), using English-Spanish bilinguals, also found asymmetrical NP in their study. Their participants showed NP from L1-to-L2, but not from L2-to-L1. Consequently, we conducted two separate $2 \times 2 \times 2$ repeatedmeasures analyses, one on the AdjRT data for the L1-to-L2 trials, and the other for the L2-to-L1 trials. For the L1-to-L2 trials, the only significant result was the main effect of NP, $F(1$, 14) $=6.60, M S E=1538, p=.022, \eta_{\mathrm{p}}{ }^{2}=.32$, indicating slower responses in the IR condition $(M=684 \mathrm{~ms})$ than in the control condition $(M=666 \mathrm{~ms})$. Paired $t$ tests were again conducted in each of the four target/distractor position conditions. None of the effects were significant, with $t(14)=1.23, p=.119, d=0.31$ in the Prime T1-Probe T1 condition; $t(14)=1.13, p=.139, d=$ 0.29 in the Prime T1-Probe D1 condition; $t(14)=0.97, p=$ $.174, d=0.25$ in the Prime D1-Probe T1 condition; and $t(14)=$ $0.69, p=.250, d=0.18$ in the Prime D1-Probe D1 condition.

For the L2-to-L1 trials, the only significant effect was the main effect of prime target position, $F(1,14)=7.37, M S E=$ 
4058, $p=.017, \eta_{\mathrm{p}}{ }^{2}=.34$, indicating longer response latencies to the probe target when the prime distractor was shown before the prime target $(721 \mathrm{~ms})$ rather than after the prime target (689 ms). Importantly, there was no evidence of NP, $F(1,14)$ $<1, n s$. Paired $t$ tests showed no significant NP effects, either, with $t(14)=0.64, p=.267, d=0.17$ in the Prime T1-Probe T1 condition; $t(14)=0.31, p=.382, d=0.08$ in the Prime T1Probe D1 condition; $t(14)=-0.98, p=.171, d=0.25$ in the Prime D1-Probe T1 condition; and $t(14)=-0.79, p=.221, d=$ 0.20 in the Prime D1-Probe D1 condition.

Consistent with previous cross-language NP research that used the traditional NP paradigm (Neumann et al., 1999), the participants in Experiment 4 showed the NP effect only in the L1-to-L2 trials. Whereas a significant NP effect was found when the prime distractor was in L1 and the probe target in L2, no evidence of NP was observed when the prime distractor was in L2 and the probe target in L1. ${ }^{4}$ In line with the inhibition account proposed by Meuter and Allport (1999), it is likely that this pattern of data reflects the different activation level between the L1 and L2 stimuli in the prime trials. Because the participants are more fluent with L1 than with L2, the baseline activation of L1 should be stronger than that of L2. When the prime distractor was in L1, strong inhibition was required to prevent it from interfering with task performance. Consequently, a robust NP effect was found. In contrast, when the prime distractor was in L2, a stimulus associated with relatively weak activation, inhibition applied to it would also be relatively weak, resulting in negligible NP.

The asymmetrical NP effects between the L1-to-L2 and the L2-to-L1 trials may thus be deemed more consistent with the inhibition account of NP effects than the episodic retrieval account (Neill \& Valdes, 1992; Neill et al., 1992). Only inhibition accounts have highlighted the importance of consistently heightened conflict between target and distractor stimuli as a major determinant of modulating NP effects (e.g., Pritchard \& Neumann, 2011; Tipper \& Cranston, 1985). Whereas both accounts can explain why a significant NP effect was found in the L1-to-L2 trials, the episodic retrieval account would have more difficulty explaining why NP disappeared in the L2-to-L1 trials, since the singularly presented distractor in the prime stream was clearly visible and not responded to (see also Fox, 1996).

In Experiments 1, 2, 3, and the L1-to-L2 trials of Experiment 4 , a robust main effect of NP was found in each experiment. However, when NP was assessed as a function of each target/ distractor position, the effect was less reliable. As this could be

\footnotetext{
${ }^{4}$ It is worth noting that in the L1-to-L2 trials, the four target stimuli differed in the number of letters that comprised the target stimuli, with the word "SIX" consisting of three letters, "FOUR" four letters, and "SEVEN" and "THREE" five letters. In theory, the participants could use the difference in word length to aid response selection, perhaps on some of the trials. Such a strategy would reduce or eliminate the NP effect. Regardless of whether such a strategy was used, the finding of the NP effect in the L1-to-L2 trials indicates the robustness of the temporal NP, which could be due to the probe distractor being a color singleton. We thank an anonymous reviewer who pointed this out.
}

due to the number of participants used in the experiments, to increase the sensitivity of detecting the NP effect, we combined the data from Experiments 1, 2, 3, and the L1-to-L2 trials of Experiment 4, and then conducted a mixed ANOVA with prime-probe relation, prime target position, and probe target position as within-subjects variables and Experiment as a betweensubject variable. Three significant main effects were found, $F(1$, 79) $=14.21, M S E=4659, p<.001, \eta_{\mathrm{p}}^{2}=.15$, for prime-probe relation; $F(1,79)=8.07, M S E=3500, p=.006, \eta_{\mathrm{p}}{ }^{2}=.09$, for prime target position, and $F(1,79)=7.46, M S E=20949, p=$ $.008, \eta_{\mathrm{p}}{ }^{2}=.09$, for probe target position. As expected, responses were faster in the control condition $(738 \mathrm{~ms})$ rather than in the IR condition $(762 \mathrm{~ms})$, when the prime target appeared at the third position $(743 \mathrm{~ms})$ rather than at the fifth position $(756 \mathrm{~ms})$, and when the probe target was at the fifth position $(735 \mathrm{~ms})$ rather than at the third position $(765 \mathrm{~ms})$. In addition, prime target position interacted with probe target position, $F(1,79)=$ $19.45, M S E=2924, p<.001, \eta_{\mathrm{p}}{ }^{2}=.20$. Whereas the difference in the AdjRT did not differ significantly between the Prime T1Probe T1 and the Prime T1-Probe D1 conditions (a difference of $-9 \mathrm{~ms}$ ), the AdjRT was significantly longer in the Prime D1Probe T1 condition than in the Prime D1-Probe D1 condition (a difference of $51 \mathrm{~ms}$ ). Tukey's HSD test showed, once again, that the AdjRT in the Prime D1-Probe T1 condition was longer than in the other three conditions. This pattern of data is consistent with the notion that it takes time to replenish used-up resources. When the targets in the prime and probe trials were close together temporally as in the Prime D1-Probe T1 condition with the prime target at the fifth position and the probe target at the third position, responses to the probe target were delayed due to insufficient attentional resources, a result consistent with the finding of AB under RSVP (Wong, 2002). Wong measured both the missing rates and the response latencies of $\mathrm{T} 2$ as a function of T1-T2 lag. RT decreased steadily from lag 2 to lag 5. These results, together with the finding of the delayed responses in the Prime D1-Probe T1 condition in the present study, underscore the temporal constraint of attentional allocation.

We again conducted a series of four $t$ tests to examine the NP effect in each target/distractor position. Significant NP effects were found in all the four conditions: $t(82)=2.84, p$ $=.003, d=0.31$, in the Prime T1-Probe T1 condition; $t(82)=$ $2.08, p=.020, d=0.23$, in the Prime T1-Probe D1 condition; $t$ $(82)=1.98, p=.026, d=0.22$, in the Prime D1-Probe T1 condition, and $t(82)=2.52, p=.007, d=0.28$, in the Prime D1-Probe D1 condition. As indicated by the value of the $d$ 's, the size of the effect was between small to medium.

\section{General discussion}

The primary goal of this article was to investigate the mechanisms of attentional selection among temporally separated stimuli in a new RSVP-NP paradigm with respect to visual 
linguistic interference control (Wong, 2012). Our findings extend Wong's in three important ways. First, they show the robustness of NP under RSVP by conceptually replicating and extending the effects with different materials and experimental setup. Second, they show that NP under RSVP can occur at semantic levels within a language, as well as with translation equivalents between languages in bilinguals. Third, the finding of stronger NP of L1-to-L2 than L2-to-L1 trials can be readily explained in the framework of distractor inhibition account (e.g., Houghton \& Tipper, 1994; Tipper, 1985) but not so in other accounts of NP including memorybased episodic retrieval accounts (e.g., Mayr \& Buchner, 2006; Neill \& Vales, 1992).

More specifically, Experiment 1 used identical stimuli for the probe target and the prime distractor. Consistent with
Wong's (2012) study, a significant NP effect was found. Experiment 2 explored semantic NP between the prime distractor and the probe target. NP was again observed, suggesting that the effect of NP under the RSVP paradigm was not limited to identity NP only. Experiment 3 required the participants to shift from one representational form to another between the prime and probe trials. Although the participants responded to a digit while having to ignore a Chinese number word in the prime trial and responded to a Chinese number word while having to ignore a digit in the probe trial, the magnitude of the NP effect did not appear to decrease much compared with that in Experiment 1. Experiment 4 investigated Chinese-English cross-language NP. A significant NP effect was found in the L1-to-L2 trials, in which the prime distractor was in the participants' L1 and the probe target in their L2. No
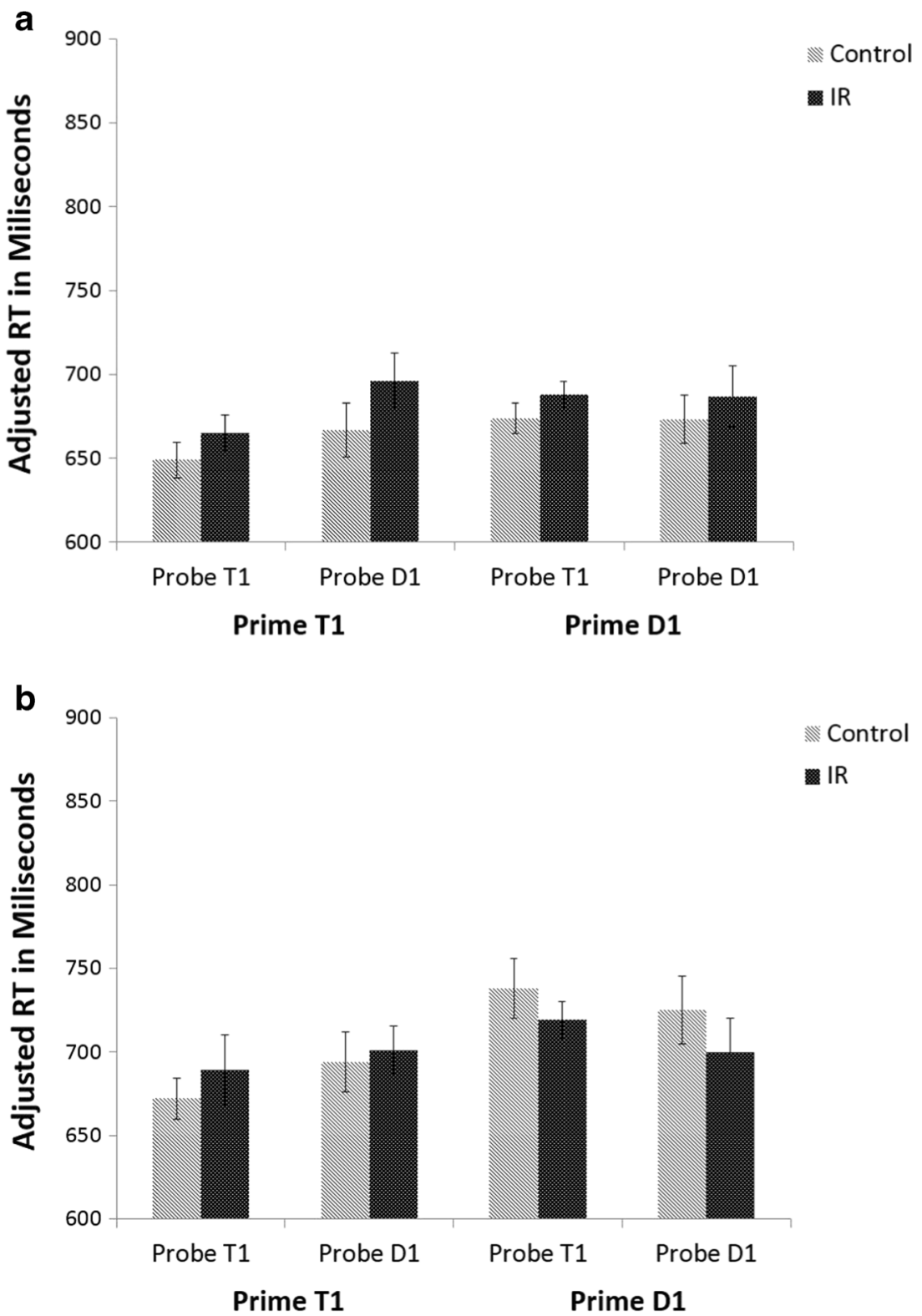

Fig. 7 (A and B). Results from Experiment 4. T1, the target appeared before the distractor. D1, the distractor appeared before the target. $R T$ reaction time 
effect was found in the L2-to-L1 trials. These results are similar to previous findings using the traditional NP paradigm, in which the target and distractor are presented simultaneously in both the prime and probe trials, suggesting that similar mechanisms may underlie the NP effects in the two paradigms.

\section{Implications for NP theories}

Four major theories have been proposed to explain the mechanisms that give rise to NP (see Frings et al., 2015; Fox, 1995; May et al., 1995; Mayr \& Buchner, 2007, and Tipper, 2001, for reviews). Before we begin our discussion, it is worth noting that although different theories emphasize the importance of different mechanisms and have different explanatory power with respect to the empirical findings from prior research and the present experiments, these theories are not necessarily exclusive of one another (Kane, May, Hasher, Rahhal, \& Stoltzfus, 1997; Neill, 2007; Tipper, 2001), nor were our experiments designed to discriminate the different theories. Below, we first describe each theory briefly. We then discuss our results in relation to the theory.

Distractor inhibition The earliest and one of the two most influential theories of NP is the distractor inhibition account, first proposed by Tipper (1985) and later revised and extended by Houghton and Tipper (1994; see Tipper, 2001, for a review). According to this account, NP is a by-product of the target selection process, during which the representation of the distractor is inhibited. Depending on the task, the inhibition can occur at a physical level or at a semantic level, and the degree of inhibition can be automatically adjusted in response to the potency of the distractor interference via feedback mechanisms. When the distractor in the prime trial becomes the target in the probe trial, the processing of the target is delayed relative to a new item. This can be caused by the lingering inhibition of the previously suppressed stimulus representation, especially when the interval between the prime and probe trials is short, or by the inhibitory processes associated with the stimulus, whose appearance as a probe target can trigger the retrieval of its prior processing episode in which the representation of the stimulus was inhibited.

The distractor inhibition account can explain the results of Experiments 1 to 3 . Inhibition was applied to the distractor when it was encountered in the RSVP stream. Even though the distractor was presented alone, as the only other stimulus in the same category as the target (i.e., a digit or a number word), it was important for its representation to be inhibited so that the target could be responded to correctly. Because the probe target and the probe distractor were either the same in physical appearance (in Experiments 1 and 3) or shared the same meaning (in Experiment 2), inhibition could be applied at the physical or semantic level or both. NP could arise because the residual inhibition to the internal presentation of the distractor was still present when the probe target appeared, or it could arise because the probe target triggered the retrieval of the inhibitory processes associated with the prime distractor.

The distractor inhibition account can also explain the asymmetry between the L1-to-L2 trials and the L2-to-L1 trials in Experiment 4. As the participants are more familiar with L1 than L2, the activation of L1 stimuli should be greater than the activation of L2 stimuli. Previous research has shown that a stimulus with greater activation is more strongly inhibited than a stimulus with weaker activation, and that NP is larger when the prime distractor is the former than the latter (Neumann et al., 1999; Wong, 2012). Given that the activation to stimuli in L1 is stronger than to stimuli in L2, greater inhibition would be applied to the prime distractor in L1-to-L2 trials than in L2to-L1 trials. Consequently, more NP was found in the former than the latter, a result consistent with the notion of NP as the result of a flexible, reactive inhibition process capable of adjusting the degree of inhibition in accordance with the amount of distractor interference in the prime trial.

Is it possible that in addition to the inhibition of the distractor representation and/or its access to the response system as proposed by Tipper and colleagues (Houghton \& Tipper, 1994; Tipper, 1985; Tipper \& Cranston, 1985), the NP effects found in the present study could also be due to the inhibition of the motor program associated with the prime distractor $?^{5}$ In our experiments, responses to the prime and probe trials involved the same motor programs (i.e., each finger was mapped to a specific stimulus, and the same mapping occurred in both the prime and probe trials). In other words, a specific response decision is bound to a specific motor program, making it impossible to know whether NP found in the IR condition was due to perceptual/decision processes, or motor programs, or both.

The motor inhibition account is consistent with the results of Experiments 1-3. However, it is difficult to explain the asymmetrical NP results found in Experiment 4 unless we assume that participants apply differential degree of motor inhibition as a function of distractor interference. In a recent study, Nett, Bröder, and Frings (2016) required their participants to use different hands to perform the task in the prime and probe trials. The results show that the participants were faster when they had to repeat, rather than change, the decision from the prime to the probe trial. They were also faster when they had to ignore the same distractor rather than a different distractor from the prime to the probe trial. Given that motor programs could not be transferred between the prime and probe trials due to the use of different hands, these results indicated that a facilitatory effect occurred independent of motor programs. In light of these results, it seems more parsimonious to explain the NP effects in terms of distractor inhibition rather than the inhibition of motor programs in the present study.

\footnotetext{
${ }^{5}$ We thank Todd Kahan for proposing this possibility.
} 
As the discussion above indicates, the NP effects found in the present study, as well as in Wong (2012), can be interpreted in the framework of the distractor inhibition theory. In addition to providing support for the theory, these studies also show that distractor representation can be inhibited quickly and effectively. Despite the rapid presentation of each item, the participants showed robust NP, suggesting that the temporal limitations typically associated with stimulus identification in RSVP experiments did not affect the manifestation of NP effects, at least in the present paradigm. Interestingly, neither the number of intervening stimuli between the prime distractor and the probe target nor the position of the distractor relative to the target in a trial (i.e., the distractor preceded or followed the target in the prime or the prime trial) affected the magnitude of NP. These results are likely due to the distractor being a color singleton. Singletons are highly salient and can attract attention involuntarily (Theeuwes, 1992; 1994). In our study, the prime distractor was always a red color singleton. A useful strategy to prevent it from interfering with the processing of the target would be to adopt an experimental-wide attentional setting that inhibits the representation of the red stimulus regardless of its temporal position in a trial. Such a strategy would be especially effective in an RSVP paradigm in which stimuli were presented very rapidly. Selectively inhibiting only those stimuli that occurred before but not after the target would be both effortful and prone to error.

Episodic retrieval The second and the other most influential theory of NP is the episodic retrieval account, which considers NP as primarily a memory phenomenon, the result of an encoding/retrieval interaction. The episodic retrieval theory, originally proposed by Neill and colleagues (Neill \& Valdes, 1992; Neill et al., 1992) and later extended by Neill and Mathis (1998), assumes that the occurrence of the probe target triggers the retrieval of the most recent episode associated with that stimulus. The retrieved episode contains the perceptual/semantic and the nonresponse information of the prime distractor. It can also contain the processing operations that occurred in the prime trial. NP is due to the delay in resolving the conflict between the response and/or the processing operations required of the probe target in the IR condition and the nonresponse information and/or the processing operations associated with the stimulus in the preceding prime trial. More recently, a stimulus-response variant of the episodic retrieval account was proposed (Mayr, Buchner, \& Dentale, 2009; Rothermund, Wentura, \& De Houwer, 2005). This account was developed from the "event file" theory proposed by Hommel (1998), who claims that the episodic representation of a stimulus contains not only the features of that stimulus but also the corresponding response and the stimulus-response binding. According to the stimulus-response account of NP, the retrieved episode contains information of the prime distractor, the prime target, and the response to the target. When the response to the prime target differs from the response to the probe target, it takes time to resolve the conflict, leading to NP in the IR condition.

In the present study, all the above accounts can explain the results of Experiments 1-3, but none predicts the results of Experiment 4. The results of Experiments 1-3 can be explained if we assume, as proposed by Neill and colleagues (Neill \& Valdes, 1992; Neill et al., 1992), that a probe target can serve as an effective retrieval cue for a prime distractor not only when the two stimuli are physically identical but also when they are conceptually similar. Whether two stimuli can be considered "conceptually similar" depends on the requirement of the task. Suppose the task is to categorize a stimulus as an animal or an object, then a monkey and a snake are conceptually similar, for both are instances of animals. In contrast, if the task is to determine whether a stimulus is a mammal or a reptile, then a monkey and a snake are not conceptually similar, for one is a mammal while the other is a reptile. In the present experiments, the participants were required to respond at a semantic level (e.g., they pressed the same response key for "FOUR" and " 4 " in Experiment 2). Consequently, a number word could cue the retrieval of the memory trace of an Arabic digit and vice versa so long as the two stimuli referred to the same numerical concept. Regardless of whether the retrieved memory trace contained the "do-not-respond" tag, the specific processing operations linked to the prime distractor, or the response made to the prime target, there was inconsistency between the retrieved information and the response/processing operations required of the probe target, resulting in NP in the IR condition.

Contrary to the results of Experiments 1-3, which fit the episodic retrieval theory quite easily, the results of Experiment 4 were hard to explain. Because the episodic retrieval account does not evoke inhibitory mechanisms, it does not distinguish stimuli on the basis of their activation level at the time of encounter. Thus, even though an L1 stimulus in Experiment 4 should elicit greater activation and therefore greater inhibition when it appeared as a distractor in the prime trial than an L2 stimulus, from the perspective of the episodic retrieval theory, the two types of trials should not differ in the ability to elicit NP. The finding of a significant NP in the L1-to-L2 trials but not in the L2-to-L1 trials in Experiment 4 is inconsistent with this prediction, and none of the versions of the episodic retrieval theory can explain this result easily.

Feature mismatching The feature mismatching theory proposed by Park and Kanwisher (1994) is the third major account of NP. The account was proposed primarily to explain NP in localization tasks. In these tasks, participants respond to the location of a pre-defined target, and the location of the probe target relative to that of the prime distractor is manipulated. In the IR condition, the probe target appears at the location previously occupied by the prime distractor. In the control condition, the location of the probe target was not occupied by any stimulus in the 
prime trial. Participants typically take longer to perform the task in the IR condition than in the control condition, and this location NP effect is interpreted as the result of a mismatch in the binding of a stimulus identity to its location between the prime and probe trials.

The feature mismatch account can explain some NP effects in localization tasks, but it cannot explain NP in those localization and identity tasks in which the binding between different stimulus features does not change from the prime to the probe trial (e.g., Milliken, Tipper, \& Weaver, 1994; Tipper \& Cranston, 1985; Tipper, Weaver, \& Milliken, 1995). For example, Tipper and Cranston (Experiment 4) asked their participants to switch response selection criterion from the prime to the probe trial by responding to a red target while ignoring a green distractor in the prime trial but responding to a green target while ignoring a red distractor in the probe trial. NP was found in the IR condition even though there was no feature mismatch between the prime and probe trials because the same green stimulus was used as both the prime distractor and the probe target. This and similar other results suggest that feature mismatching is unlikely to be the primary mechanism that gives rise to NP.

With respect to the present study, the feature mismatch theory can explain the results of Experiments 1-3, but not the asymmetrical NP effects between the L1-to-L2 and the L2-to-L1 trials in Experiment 4. The degree of feature mismatch between color and identity from the prime to the probe trial was the same in both the L1-to-L2 and L2-to-L1 trials. Thus, if NP were caused primarily by the binding of different features in the prime and probe trials, our participants would have shown comparable magnitude of NP in the two types of trials in Experiment 4 instead of a larger NP effect in the L1-to-L2 trials.

Temporal discrimination The fourth major theory of NP is the temporal discrimination account originally proposed by Milliken and colleagues (Milliken \& Joordens, 1996; Milliken, Joordens, Merikel, \& Seiffert, 1998; see also Frings \& Eder, 2009; Healy \& Burt, 2003), which considers NP as the result of a delay in categorizing the probe target as being "old" or "new". According to this account, when a stimulus is encountered and categorized as "new," perceptual analysis is performed, and a relatively fast response is then made. When a stimulus is encountered and categorized as "old," the previous response to the stimulus is retrieved, resulting in a very fast response because there is no need for perceptual analysis. In situations when there is uncertainty about the status of a stimulus as being "old" or "new," categorizing the stimulus would take longer, resulting in a delay in response. In the IR condition, the probe target was the ignored distractor in the preceding trial. This prevents the probe target from being categorized quickly, for it is neither familiar enough to warrant a quick "old" judgment nor unfamiliar enough to warrant a quick "new" judgement. Resolving the uncertainty takes time, leading to NP.

Similar to the episodic retrieval and feature mismatch accounts, the temporal discrimination account can explain the results of Experiments 1-3, but not the findings of Experiment 4. The degree of ambiguity in categorizing a probe target as an "old" or "new" stimulus when its translational equivalent served as a prime distractor should be comparable regardless of whether the prime distractor was an L1 or an L2 stimulus. The finding of a significant NP effect in the L1-to-L2 trials but not in the L2-to-L1 trials is therefore inconsistent with the prediction of the temporal discrimination account.

Summary Of the four theories of NP, our results as a whole are best explained in the framework of the distractor inhibition account. However, it is important to note that just because the results of Experiment 4 in the present study cannot be easily accounted for by the other three theories of NP, it does not mean that the mechanisms proposed by these theories do not apply to NP in the present study or in an RSVP-NP paradigm in general. On the contrary, it is highly likely that multiple mechanisms contributed to the observed NP effects in the present study, especially in Experiments 1-3, and that the mechanisms that give rise to NP are the same or very similar in both the traditional NP paradigm and the RSVP-NP paradigm developed by Wong (2012).

\section{Potential relevance for $\mathrm{AB}$ phenomena}

Before any parallels can be drawn between $\mathrm{AB}$ and the present task, it would first be necessary to establish that a red non-target in the T2 position (which is apt to capture attention due to its color singleton status) is susceptible to a decrement in reporting (i.e., an $\mathrm{AB}$ effect). If such a stimulus then turned out indeed to be susceptible to $A B$, it would be necessary to pursue why both preand post-target nontaget distractors produce NP effects in the present context. In light of the recommendation by Dux and Marios (2009), it might be best to pursue this issue using reaction-time versions of $\mathrm{AB}$ tasks (e.g., Wong, 2002), rather than a delayed recall. One thing that is clear is that in both tasks non-reported items in the T2 position in RSVP streams are processed conceptually, because they can produce subsequent semantic priming effects. It is well known in the negative priming literature that the more conflict (or anticipated conflict) between target and distractor stimuli the greater the magnitude of NP seems to be. In accordance with this literature, the idea is that the mental representation of non-target stimuli must initially be activated, otherwise there is no signal for inhibition to home in on (Neumann \& DeSchepper, 1991). It could be that in AB tasks where subsequent positive priming is observed from blinked T2 items that they are nevertheless processed to a high level, and their mental representations are only weakly inhibited so they remain relatively activated, and are thereby capable of producing conceptual facilitatory priming. It should be noted that we are not using the terms "inhibition" and "suppression" to indicate any kind of blocking or gating as they are used in the $\mathrm{AB}$ literature. We are using these terms the way they are used in the negative 
priming literature (i.e., indicative of a late-selection active inhibition after initial activation of a non-target distractor stimulus).

In our task, the pre- and post-target red distractors are always unwanted potentially conflicting stimuli with no requirement to remember them. Such stimuli are rife for undergoing active inhibition in order to eliminate interference with the target stimulus. In both the AB and NP cases, the outcome may be a function of how attention works in terms of modulating mental representations in order to resolve conflict with the momentary goals of the task at hand. In this scenario, selective attention acts to minimize the interference posed by any potentially distracting stimuli whether they appear concurrently or in a temporally separated fashion. In the dualtarget AB task, the T2 may momentarily be weakly inhibited to avoid producing interference with the higher priority $\mathrm{T} 1$, while remaining activated enough to produce positive priming. Such late-selection attentional modulation involving degrees of inhibitory control suggests a shared mechanism responsible for both $\mathrm{AB}$ positive priming effects and the NP phenomena reported here. Future research should address this possibility, because it could be a pathway toward integrating what seem to be vastly disparate findings.

\section{Summary, recommendations, and conclusion}

In four experiments, we explored target selection among temporally separated stimuli. Taken together, the results suggest that the mechanisms that underlie temporal attentional selection in NP under RSVP may be similar, if not identical, to those that underlie contemporaneous attentional selection with concurrently presented target and distractor stimuli in more traditional NP paradigms. Gaining a fuller understanding of the mechanism(s) underpinning NP phenomena remains an important goal for understanding selective attention more broadly (Fox, 1995; Frings et al., 2015). The findings in the present study confirm the suitability of the RSVP-NP methodology by demonstrating the generalizability of NP phenomena in selective attention. In these regards, two recommendations about using the traditional NP paradigms may also provide a cogent way forward using the RSVP-NP methodology.

Firstly, Christie and Klein (2008) posited that using the full set of all possible prime-probe target-distractor relationships would be most promising for fleshing out the full set of mechanisms underpinning negative priming phenomena (see Neumann \& Deschepper, 1991; Stadler \& Hogan, 1996, for examples of these manipulations). They pointed out that compared with a control condition, in which there is no relationship between the target and distractor stimuli in prime and probe couplets, systematic degrees of reaction-time costs and benefits in processing are produced by the remaining six conditions. Along with the typical IR condition, for example, a significant cost is also produced if the prime target becomes the probe distractor, and an even greater cost is produced if the prime distractor becomes the probe target, combined with prime target becoming the probe distractor. On the other hand, a benefit in processing is observed in an attended repetition condition in which the target in the prime becomes the target in the probe. A similar benefit emerges if the prime distractor repeats as the probe distractor, and an even greater benefit is observed if both the target repeats as the subsequent target, combined with the prime distractor becoming the probe distractor. Each of these combinations of conditions can easily be adapted to the RSVPNP procedure by using prime and probe streams each containing temporally separated target and distractor stimuli encompassing these same relationships. Because the findings from experiments that have used these seven conditions have been instrumental in refuting certain explanations of NP (see e.g., Christie \& Klein, 2008; Frings \& Wühr, 2007), they should be used more frequently not only in the more traditional NP paradigms, but also in the RSVP-NP paradigm.

Secondly, Henson, Eckstein, Waszak, Frings, and Horner (2014) recently observed that people can rapidly form arbitrary associations between stimuli and the covert responses they make in the presence of those stimuli, including such responses to the non-target prime distractors in traditional NP tasks. To avoid the consequences of such stimulus-response

(S-R) bindings in priming, they recommend using a large pool of stimuli, combined with naming or perceptual identification on the prime, for example, together with a different task such as classification on the probe. Under these circumstances each stimulus would be associated with a unique response that is not repeated in the probe component of a trial, and so could not modulate priming (see Neumann, et al., 1999, for an implementation of such a task using naming followed by a lexical decision task with a large pool of stimuli). If Henson et al. are correct, the priming effects reported by Neumann et al. should be independent of contamination by S-R binding, because stimulus cued responses in the probe do not overlap with previous responses to those stimuli in the prime. From our perspective, these recommendations should be incorporated in investigations that further build on the types of manipulations that could be adopted in new variants of the RSVP-NP task. By doing so, unintended artefacts can be eliminated thereby yielding more straightforward results and potentially more reliable interpretations. Further insights about the role of selective attention and other fundamental aspects of cognition are sure to be gained by implementing the recommendations made by Christie and Klein (2008) and Henson et al. in future instantiations of RSVP-NP tasks.

Acknowledgements We thank Kin Fai Ellick Wong, Liana Machado, Todd Kahan, and three anonymous reviewers for their helpful comments on a previous version of the manuscript. 


\section{Appendi A}

Table 1 Adjusted reaction times in milliseconds as a function of the stimulus position in the prime and probe trials, and the prime-probe relationship in Experiments 1-3

\begin{tabular}{|c|c|c|c|c|}
\hline \multirow[b]{3}{*}{ Stimulus position in prime trial } & \multicolumn{4}{|c|}{ Stimulus position in probe trial } \\
\hline & \multicolumn{2}{|l|}{ Probe T1 } & \multicolumn{2}{|l|}{ Probe D1 } \\
\hline & Control & IR & Control & IR \\
\hline \multicolumn{5}{|l|}{ Experiment 1} \\
\hline Prime T1 & $762(16.3)$ & $797(14.2)$ & 769 (11.6) & $791(17.7)$ \\
\hline Prime D1 & $795(13.4)$ & $827(13.2)$ & 747 (11.9) & $788(18.1)$ \\
\hline \multicolumn{5}{|l|}{ Experiment 2} \\
\hline Prime T1 & $795(21.7)$ & $831(18.4)$ & $729(13.0)$ & $750(18.8)$ \\
\hline Prime D1 & $858(20.9)$ & $842(17.9)$ & $748(22.2)$ & $774(24.2)$ \\
\hline \multicolumn{5}{|l|}{ Experiment 3} \\
\hline Prime T1 & $689(13.6)$ & $718(15.8)$ & $691(11.9)$ & $709(17.2)$ \\
\hline Prime D1 & $737(14.9)$ & $755(12.8)$ & $667(11.2)$ & $672(13.7)$ \\
\hline
\end{tabular}

Within-subjects standard errors are in parentheses

$T 1$ the target appeared before the distractor, $D 1$ the distractor appeared before the target, $I R$ ignored repetition condition

\section{Appendix B}

Table 2 Mean reaction times and error rates as a function of the stimulus position in the prime and probe trials, and the prime-probe relationship in Experiments 1-3

\begin{tabular}{|c|c|c|c|c|}
\hline \multirow[b]{3}{*}{ Stimulus position in prime trial } & \multicolumn{4}{|c|}{ Stimulus position in probe trial } \\
\hline & \multicolumn{2}{|l|}{ Probe $\mathrm{T} 1$} & \multicolumn{2}{|l|}{ Probe D1 } \\
\hline & Control & IR & Control & IR \\
\hline \multicolumn{5}{|l|}{ Experiment 1} \\
\hline \multicolumn{5}{|l|}{ Reaction times (ms) } \\
\hline Prime T1 & $668(6.2)$ & $688(6.8)$ & $639(7.6)$ & $639(7.5)$ \\
\hline Prime D1 & $694(6.9)$ & $715(8.7)$ & $623(6.0)$ & $650(6.6)$ \\
\hline \multicolumn{5}{|l|}{ Error rates ( $\%$ incorrect) } \\
\hline Prime T1 & $10.6(1.0)$ & $12.4(0.9)$ & $15.6(0.7)$ & $17.0(1.0)$ \\
\hline Probe D1 & $11.4(1.0)$ & $12.5(1.0)$ & $14.6(1.0)$ & $15.2(0.9)$ \\
\hline \multicolumn{5}{|l|}{ Experiment 2} \\
\hline \multicolumn{5}{|l|}{ Reaction times (ms) } \\
\hline Prime T1 & $696(8.8)$ & $721(10.0)$ & $635(7.4)$ & $631(10.8)$ \\
\hline Prime D1 & $745(9.1)$ & $722(9.3)$ & $651(10.3)$ & $649(9.4)$ \\
\hline \multicolumn{5}{|l|}{ Error rates ( $\%$ incorrect) } \\
\hline Prime T1 & $11.7(1.7)$ & $12.9(1.5)$ & $12.9(1.2)$ & $15.4(1.2)$ \\
\hline Probe D1 & $12.5(1.5)$ & $13.8(1.3)$ & $12.1(1.6)$ & $15.3(1.7)$ \\
\hline \multicolumn{5}{|l|}{ Experiment 3} \\
\hline \multicolumn{5}{|l|}{ Reaction times (ms) } \\
\hline Prime T1 & $660(10.8)$ & $682(11.4)$ & $637(9.4)$ & $645(10.9)$ \\
\hline Prime D1 & $694(10.3)$ & $712(9.5)$ & $635(8.3)$ & $633(10.6)$ \\
\hline \multicolumn{5}{|l|}{ Error rates (\% incorrect) } \\
\hline Prime T1 & $3.9(0.8)$ & $4.8(0.8)$ & $7.9(0.8)$ & $8.8(1.1)$ \\
\hline Probe D1 & $5.5(0.9)$ & $5.4(0.8)$ & $4.9(0.6)$ & $5.9(0.9)$ \\
\hline
\end{tabular}

Within-subjects standard errors are in parentheses

$T 1$ the target appeared before the distractor, $D 1$ the distractor appeared before the target, $I R$ ignored repetition condition 
Table 3 Adjusted reaction times in milliseconds as a function of language, the stimulus position in the prime and probe trials, and the prime-probe relationship in Experiment 4

\begin{tabular}{|c|c|c|c|c|}
\hline \multirow[b]{3}{*}{ Stimulus position in prime trial } & \multicolumn{4}{|c|}{ Stimulus position in probe trial } \\
\hline & \multicolumn{2}{|l|}{ Probe T1 } & \multicolumn{2}{|l|}{ Probe D1 } \\
\hline & Control & IR & Control & IR \\
\hline \multicolumn{5}{|l|}{ L1 to L2 Trials } \\
\hline Prime T1 & $649(10.5)$ & $665(11.0)$ & $667(16.1)$ & $696(16.9)$ \\
\hline Prime D1 & $674(9.0)$ & $688(7.9)$ & $673(14.4)$ & $687(18.3)$ \\
\hline \multicolumn{5}{|l|}{ L2 to L1 Trials } \\
\hline Prime T1 & $672(12.3)$ & $689(21.3)$ & $694(18.1)$ & $701(14.6)$ \\
\hline Prime D1 & $738(17.7)$ & $719(11.2)$ & $725(20.0)$ & $700(20.1)$ \\
\hline
\end{tabular}

Within-subjects standard errors are in parentheses

$T 1$ the target appeared before the distractor, $D 1$ the distractor appeared before the target, $I R$ ignored repetition condition

Table 4 Mean reaction times and error rates as a function of language, the stimulus position in the prime and probe trials, and the prime-probe relationship in Experiment 4

\begin{tabular}{|c|c|c|c|c|}
\hline \multirow[b]{3}{*}{ Stimulus position in prime trial } & \multicolumn{4}{|c|}{ Stimulus position in probe trial } \\
\hline & \multicolumn{2}{|l|}{ Probe T1 } & \multicolumn{2}{|l|}{ Probe D1 } \\
\hline & Control & IR & Control & IR \\
\hline \multicolumn{5}{|l|}{ L1 to L2 Trials } \\
\hline \multicolumn{5}{|l|}{ Reaction times (ms) } \\
\hline Prime T1 & $631(9.0)$ & $642(9.0)$ & $630(11.8)$ & $661(14.8)$ \\
\hline Prime D1 & $652(9.0)$ & $668(8.2)$ & $635(11.1)$ & $639(9.5)$ \\
\hline \multicolumn{5}{|l|}{ Error rates (\% incorrect) } \\
\hline Prime T1 & $2.7(0.7)$ & $3.2(1.1)$ & $5.5(1.3)$ & $5.0(1.0)$ \\
\hline Probe D1 & $3.1(1.0)$ & $2.7(1.0)$ & $4.8(1.6)$ & $6.8(1.5)$ \\
\hline \multicolumn{5}{|l|}{ L2 to L1 Trials } \\
\hline \multicolumn{5}{|l|}{ Reaction times (ms) } \\
\hline Prime T1 & $642(9.0)$ & $653(12.3)$ & $630(9.9)$ & $643(10.6)$ \\
\hline Prime D1 & $690(14.4)$ & $692(10.5)$ & $653(11.7)$ & $627(10.4)$ \\
\hline \multicolumn{5}{|l|}{ Error rates (\% incorrect) } \\
\hline Prime $\mathrm{T} 1$ & $4.2(0.9)$ & $5.0(1.3)$ & $8.9(1.5)$ & $7.6(1.9)$ \\
\hline Probe D1 & $6.4(1.1)$ & $3.9(0.7)$ & $9.3(1.8)$ & $9.7(2.0)$ \\
\hline
\end{tabular}

Within-subjects standard errors are in parentheses

$T 1$ the target appeared before the distractor, $D 1$ the distractor appeared before the target, $I R$ ignored repetition condition 


\section{References}

Cerf, M., Thiruvengadam, N., Mormann, F., Kraskov, A., Quiroga, R. Q., Koch, C., \& Fried, I. (2010). On-line, voluntary control of human temporal lobe neurons. Nature, 467, 1104-1108.

Chambers, C. D., Stokes, M. G., \& Mattingley, J. B. (2004). Modalityspecific control of strategic spatial attention in parietal cortex. Neuron, 44, 925-930.

Chen, Z. (2003). Attentional focus, processing load, and Stroop interference. Perception \& Psychophysics, 65, 888-900.

Christie, J. J., \& Klein, R. M. (2008). On finding negative priming from distractors. Psychonomic Bulletin \& Review, 15, 866-873.

Chun, M. M., \& Potter, M. C. (1995). A two-stage model for multiple target detection in rapid serial visual presentation. Journal of Experimental Psychology: Human Perception and Performance, 21, 109-127.

Chun, M. M., \& Wolfe, J. M. (1996). Just say no: How are visual searches terminated when there is no target present? Cognitive Psychology, 30, 39-78.

Cousineau, D. (2005). Confidence intervals in within-subject designs: A simpler solution to Loftus and Masson's method. Tutorials in Quantitative Methods for Psychology, 1, 42-45.

D'Angelo, M., Thomson, D. R., Tipper, S. P., \& Milliken, B. (2016). Negative priming 1985 to 2015: A measure of inhibition, the emergence of alternative accounts, and the multiple process challenge. The Quarterly Journal of Experimental Psychology, 69, 1890-1909.

De Jong, R. (1995). Strategical determinants of compatibility effects with task uncertainty. Acta Psychologica, 88, 187-207.

Driver, J., \& Tipper, S. P. (1989). On the nonselectivity of 'selective' seeing: Contrasts between interference and priming in selective attention. Journal of Experimental Psychology: Human Perception and Performance, 15, 304-314.

Duscherer, K., \& Holender, D. (2002). No negative semantic priming from unconscious flanker words in sight. Journal of Experimental Psychology: Human Perception and Performance, 28, 839-853.

Dux, P. E., Coltheart, V., \& Harris, I. M. (2006). On the fate of distractor stimuli in rapid serial visual presentation. Cognition, 99, 355-382.

Dux, P. E., \& Marois, R. (2009). How humans search for targets through time: A review of data and theory from the attentional blink. Attention, Perception, \& Psychophysics, 71, 1683-1700.

Filippi, R., Karaminis, T., \& Thomas, M. S. C. (2014). Language switching in bilingual production: Empirical data and computational modelling. Bilingualism: Language and Cognition, 17, 294-315.

Fleck, M. S., \& Mitroff, S. R. (2007). Rare targets are rarely missed in correctable search. Psychological Science, 18, 943-947.

Fox, E. (1995). Negative priming from ignored distractors in visual selection - A review. Psychonomic Bulletin \& Review, 2, 145-173.

Fox, E. (1996). Cross-language priming from ignored words: Evidence from a common representational system in bilinguals. Journal of Memory \& Language, 2, 337-339.

Frings, C., \& Eder, A. B. (2009). The time-course of masked negative priming. Experimental Psychology, 56, 301-306.

Frings, C., Schneider, K., \& Fox, E. (2015). The negative priming paradigm: An update and implications for selective attention. Psychonomic Bulletin \& Review, 22, 1577-1597.

Frings, C., \& Wühr, P. (2007). On distractor-repetition benefits in the negative-priming paradigm. Visual Cognition, 15, 166-178.

Harris, I., Benito, C., \& Dux, P. (2010). Priming from distractors in rapid serial visual presentation is modulated by image properties and attention. Journal of Experimental Psychology: Human Perception and Performance, 36, 1595-1608.

Harris, I. M., \& Little, M. J. (2010). Priming the semantic neighbourhood during the attentional blink. PLoS One, 5, e12645.
Harvey, N. (1984). The Stroop effect: Failure to focus attention or failure to maintain focusing? The Quarterly Journal of Experimental Psychology. A, 36, 89-115.

Healy, D., \& Burt, J. S. (2003). Attending to the distractor and old/new discrimination in negative priming. The Quarterly Journal of Experimental Psychology A, 56, 421-443.

Henson, R. N., Eckstein, D., Waszak, F., Frings, C., \& Horner, A. J. (2014). Stimulus-response bindings in priming. Trends in Cognitive Sciences, 18, 376-384.

Hommel, B. (1998). Event files: Evidence for automatic integration of stimulus-response episodes. Visual Cognition, 5, 183-216.

Houghton, G., \& Tipper, S. P. (1994). A model of inhibitory mechanisms in selective attention. In I. D. Dagenbach \& T. H. Carr (Eds.), Inhibitory processes in attention, memory, and language (pp. 53112). San Diego: Academic Press.

Houghton, G., Tipper, S. P., Weaver, B., \& Shore, D. I. (1996). Inhibition and interference in selective attention: Some tests of a neural network model. Visual Cognition, 3, 119-164.

Hutchison, K. A. (2002). The effect of asymmetrical association on positive and negative semantic priming. Memory \& Cognition, 30, 1263-1276.

Kane, M. J., May, C. P., Hasher, L., Rahhal, T., \& Stoltzfus, E. R. (1997). Dual mechanisms of negative priming. Journal of Experimental Psychology: Human Perception and Performance, 23, 632-650.

Kanwisher, N. (1987). Repetition blindness: Type recognition without token individuation. Cognition, 27, 117-143.

Kiesel, A., Steinhauser, M., Wendt, M., Falkenstein, M., Jost, K., Philipp, A., \& Koch, I. (2010). Control and interference in task switching - a review. Psychological Bulletin, 136, 849-874.

Koelewijn, T., Van der Burg, E., Bronkhorst, A., \& Theeuwes, J. (2008). Priming $\mathrm{T} 2$ in a visual and auditory attentional blink task. Perception \& Psychophysics, 70, 658-666.

Lammertyn, J., \& Fias, W. (2005). Negative priming with numbers: No evidence for a semantic locus. Quarterly Journal of Experimental Psychology Section a-Human Experimental Psychology, 58, 11531172.

Luck, S. J., Vogel, E. K., \& Shapiro, K. L. (1996). Word meaning can be accessed but not reported during the attentional blink. Nature, 383, 616-618.

Macizo, P., Bajo, T., \& Paolieri, D. (2012). Language switching and language competition. Second Language Research, 28, 131-149.

MacLeod, C. M., Chiappe, D. L., \& Fox, E. (2002). The crucial roles of stimulus matching and stimulus identity in negative priming. Psychonomic Bulletin \& Review, 9, 521-528.

Maki, W. S., Frigen, K., \& Paulson, K. (1997). Associative priming by targets and distractors during rapid serial visual presentation: Does word meaning survive the attentional blink? Journal of Experimental Psychology: Human Perception and Performance, 23, 1014-1034.

Martens, S., Wolters, G., \& van Raamsdonk, M. (2002). Blinks of the mind: Memory effects of attentional processes. Journal of Experimental Psychology: Human Perception and Performance, $28,1275-1287$.

May, C. P., Kane, M. J., \& Hasher, L. (1995). Determinants of negative priming. Psychological Bulletin, 118, 35-54.

Mayr, S., Buchner, A., \& Dentale, S. (2009). Prime retrieval of motor responses in negative priming. Journal of Experimental Psychology: Human Perception and Performance, 35, 408-423.

Meuter, R. F. I., \& Allport, A. (1999). Bilingual language switching in naming: Asymmetrical costs of language selection. Journal of Memory and Language, 40, 25-40.

Milliken, B., \& Joordens, S. (1996). Negative priming without overt prime selection. Canadian Journal of Experimental Psychology, $50,333-346$ 
Milliken, B., Joordens, S., Merikle, P. M., \& Seiffert, A. E. (1998). Selective attention: A reevaluation of the implications of negative priming. Psychological Review, 105, 203-229.

Milliken, B., Tipper, S. P., \& Weaver, B. (1994). Negative priming in a spatial localization task: Feature mismatching and distractor inhibition. Journal of Experimental Psychology: Human Perception \& Performance, 20, 624-646.

Neill, W. T. (1977). Inhibitory and facilitatory processes in selective attention. Journal of Experimental Psychology-Human Perception and Performance, 3, 444-450.

Neill, W. T. (2007). Mechanisms of transfer-inappropriate processsing. In D. S. Forfein \& C. M. MacLeod (Eds.), Inhibition in cognition (pp. 63-78). Washington, DC: American Psychological Association.

Neill, W. T., \& Mathis, K. M. (1998). Negative priming and related phenomena. Psychology of learning and motivation: Advances in research and theory, 38, 1044.

Neill, W. T., \& Valdes, L. A. (1992). The persistence of negative priming: Steady-state or decay? Journal of Experimental Psychology: Learning, Memory, \& Cognition, 18, 565-576.

Neill, W. T., Valdes, L. A., Terry, K. M., \& Gorfein, D. S. (1992). Persistence of negative priming: II. Evidence for episodic trace retrieval. Journal of Experimental Psychology: Learning, Memory, and Cognition, 18, 993-1000.

Nett, N., Bröder, A., \& Frings, C. (2016). Distractor -based stimulusresponse bindings retrieve decisions independent of motor programs. Acta Psychologica, 171, 57-64.

Neumann, E., Cherau, J. F., Hood, K. L., \& Steinnagel, S. L. (1993). Does inhibition spread in a manner analogous to spreading activation? Memory, 1, 81-105.

Neumann, E., \& Deschepper, B. G. (1991). Costs and benefits of target activation and distractor inhibition in selective attention. Journal of Experimental Psychology-Learning Memory and Cognition, 17, $1136-1145$.

Neumann, E., \& DeSchepper, B. G. (1992). An inhibition-based fan effect: Evidence for an active suppression mechanism in selective attention. Canadian Journal of Psychology, 46, 1-40.

Neumann, McCloskey, M. S., \& Felio, A. C. (1999). Cross-language positive priming disappears, negative priming does not: Evidence for two sources of selective inhibition. Memory \& Cognition, 27, 1051-1063.

Park, J., \& Kanwisher, N. (1994). Negaitve priming for spatial locations: Identity mismatching, not distractor inhibition. Journal of Experimental Psychology: Human Perception and Performance, 20, 613-623.

Peirce, J. W. (2007). PsychoPy - Psychophysics software in Python. Journal of Neuroscience Methods, 162, 8-13.

Pesciarelli, F., Kutas, M., Dell'Acqua, R., Peressotti, F., Job, R., et al. (2007). Semantic and repetition priming within the attentional blink: An event-related brain potential (ERP) investigation study. Biological Psychology, 76, 21-30.

Potter, M. C. (1993). Very short-term conceptual memory. Memory \& Cognition, 21, 156-161.

Pritchard, V. E., \& Neumann, E. (2009). Avoiding the potential pitfalls of using negative priming tasks in developmental studies: Assessing inhibitory control in children, adolescents, and adults. Developmental Psychology, 45, 272-283.

Pritchard, V. E., \& Neumann, E. (2011). Classic stroop negative priming effects for children and adults diverge with less-conflicting and nonconflicting conditions. American Journal of Psychology, 124, 405419.
Raymond, J. E., Shapiro, K. L., \& Arnell, K. M. (1992). Temporary suppression of visual processing in an RSVP task: An attentional blink? Journal of Experimental Psychology: Human Perception and Performance, 18, 849-860.

Schooler, C., Neumann, E., Caplan, L. J., \& Roberts, B. R. (1997a). A time course analysis of Stroop interference and facilitation: Comparing normal individuals and individuals with schizophrenia. Journal of Experimental Psychology: General, 126, 19-36.

Schooler, C., Neumann, E., Caplan, L. J., \& Roberts, B. R. (1997b). Stroop theory, memory, prefrontal cortical functioning: Reply to Cohen et al. (1997). Journal of Experimental Psychology: General, 126, 42-44.

Shapiro, K. L., Driver, J., Ward, R., \& Sorensen, R. E. (1997). Priming from the attentional blink: A failure to extract visual tokens but not visual types. Psychological Science, 8, 95-100.

Stadler, M. A., \& Hogan, M. E. (1996). Varieties of positive and negative priming. Psychonomic Bulletin \& Review, 3, 87-90.

Stroop, J. R. (1935). Studies of interference in serial verbal reactions. Journal of Experimental Psychology, 18, 643-662.

Theeuwes, J. (1992). Perceptual selectivity for color and form. Perception \& Psychophysics, 51, 599-606.

Theeuwes, J. (1994). Stimulus-driven capture and attentional set: Selective search for color and visual abrupt onsets. Journal of Experimental Psychology: Human Perception \& Performance, 20, 799-806.

Tipper. (1985). The Negative Priming Effect - Inhibitory priming by ignored objects. Quarterly Journal of Experimental Psychology Section a-Human Experimental Psychology, 37, 571-590.

Tipper. (2001). Does negative priming reflect inhibitory mechanisms? A review and integration of conflicting views. Quarterly Journal of Experimental Psychology Section a-Human Experimental Psychology, 54, 321-343.

Tipper, \& Cranston, M. (1985). Selective attention and priming: Inhibitory and facilitatory effects of ignored primes. The Quarterly Journal of Experimental Psychology, 37, 591-611.

Tipper, S. P., \& Driver, J. (1988). Negative priming between pictures and words in a selective attention task - Evidence for semantic processing of ignored stimuli. Memory \& Cognition, 16, 64-70.

Tipper, S. P., Weaver, G., \& Milliken, B. (1995). Spatial negative priming without mismatching: Comments on park \& Kanwisher. 1994: Journal of Experimental Psychology: Human Perception \& Performance, 21, 1220-1229.

Torvalds, L. (1997). Linux: a Portable Operating System (Master's thesis, University of Helsinki, Helsinki, Finland).

Townsend, J. T., \& Ashby, F. G. (1983). Stochastic modeling of elementary psychological processes. CUP Archive.

Wong, K. F. E. (2002). The relationship between attentional blink and psychological refractory period. Journal of Experimental Psychology: Human Perception \& Performance, 28, 54-71.

Wong, K. F. E. (2012). Negative priming under rapid serial visual presentation. Plos One, 7, e37023.

Wong, K. F. E., \& Chen, H.-C. (2009). Forward and backward repetition blindness in speed and accuracy. Journal of Experimental Psychology: Human Perception \& Performance, 35, 778-786.

Wyatt, N., \& Machado, L. (2013a). Distractor inhibition: Principles of operation during selective attention. Journal of Experimental Psychology: Human Perception and Performance, 39, 245-256.

Wyatt, N., \& Machado, L. (2013b). Evidence inhibition responds reactively to the salience of distracting information during focused attention. PloS One, 8, e62809. 